\title{
Application of Bipolar Fuzzy Sets in Graph Structures
}

\author{
Muhammad Akram and Rabia Akmal \\ Department of Mathematics, University of the Punjab, New Campus, Lahore 54590, Pakistan \\ Correspondence should be addressed to Muhammad Akram; m.akram@pucit.edu.pk
}

Received 27 November 2015; Revised 25 December 2015; Accepted 28 December 2015

Academic Editor: Baoding Liu

Copyright (C) 2016 M. Akram and R. Akmal. This is an open access article distributed under the Creative Commons Attribution License, which permits unrestricted use, distribution, and reproduction in any medium, provided the original work is properly cited.

A graph structure is a useful tool in solving the combinatorial problems in different areas of computer science and computational intelligence systems. In this paper, we apply the concept of bipolar fuzzy sets to graph structures. We introduce certain notions, including bipolar fuzzy graph structure (BFGS), strong bipolar fuzzy graph structure, bipolar fuzzy $N_{i}$-cycle, bipolar fuzzy $N_{i}$-tree, bipolar fuzzy $N_{i}$-cut vertex, and bipolar fuzzy $N_{i}$-bridge, and illustrate these notions by several examples. We study $\phi$-complement, self-complement, strong self-complement, and totally strong self-complement in bipolar fuzzy graph structures, and we investigate some of their interesting properties.

\section{Introduction}

Concepts of graph theory have applications in many areas of computer science including data mining, image segmentation, clustering, image capturing, and networking. A graph structure, introduced by Sampathkumar [1], is a generalization of undirected graph which is quite useful in studying some structures including graphs, signed graphs, and graphs in which every edge is labeled or colored. A graph structure helps to study the various relations and the corresponding edges simultaneously.

A fuzzy set, introduced by Zadeh [2], gives the degree of membership of an object in a given set. Zhang [3] initiated the concept of a bipolar fuzzy set as a generalization of a fuzzy set. A bipolar fuzzy set is an extension of fuzzy set whose membership degree range is $[-1,1]$. In a bipolar fuzzy set, the membership degree 0 of an element means that the element is irrelevant to the corresponding property, the membership degree $(0,1]$ of an element indicates that the element somewhat satisfies the property, and the membership degree $[-1,0)$ of an element indicates that the element somewhat satisfies the implicit counterproperty. Kauffman defined in [4] a fuzzy graph. Rosenfeld [5] described the structure of fuzzy graphs obtaining analogs of several graph theoretical concepts. Bhattacharya [6] gave some remarks on fuzzy graphs. Several concepts on fuzzy graphs were introduced by Mordeson et al. [7]. Dinesh [8] introduced the notion of a fuzzy graph structure and discussed some related properties. Akram et al. [9-13] have introduced bipolar fuzzy graphs, regular bipolar fuzzy graphs, irregular bipolar fuzzy graphs, antipodal bipolar fuzzy graphs, and bipolar fuzzy hypergraphs. In this paper, we introduce the certain notions including bipolar fuzzy graph structure (BFGS), strong bipolar fuzzy graph structure, bipolar fuzzy $N_{i}$-cycle, bipolar fuzzy $N_{i}$-tree, bipolar fuzzy $N_{i}$-cut vertex, and bipolar fuzzy $N_{i}$-bridge and illustrate these notions by several examples. We present $\phi$-complement, self-complement, strong selfcomplement, and totally strong self-complement in bipolar fuzzy graph structures, and we investigate some of their interesting properties.

We have used standard definitions and terminologies in this paper. For other notations, terminologies, and applications not mentioned in the paper, the readers are referred to $[1,5,7,14-18]$.

\section{Preliminaries}

In this section, we review some definitions that are necessary for this paper.

A graph structure $G^{*}=\left(U, E_{1}, E_{2}, \ldots, E_{k}\right)$ consists of a nonempty set $U$ together with relations $E_{1}, E_{2}, \ldots, E_{k}$ on $U$, which are mutually disjoint such that each $E_{i}$ is irreflexive and symmetric. If $(u, v) \in E_{i}$ for some $i, 1 \leq i \leq k$, we call it an $E_{i}$-edge and write it as "uv." A graph structure 
$G^{*}=\left(U, E_{1}, E_{2}, \ldots, E_{k}\right)$ is complete, if (i) each edge $E_{i}, 1 \leq$ $i \leq k$, appears at least once in $G^{*}$; (ii) between each pair of vertices $u v$ in $U, u v$ is an $E_{i}$-edge for some $i, 1 \leq i \leq k$. A graph structure $G^{*}=\left(U, E_{1}, E_{2}, \ldots, E_{k}\right)$ is connected, if the underlying graph is connected. In a graph structure, $E_{i}$-path between two vertices $u$ and $v$, is the path which consists of only $E_{i}$-edges for some $i$, and similarly, $E_{i}$-cycle is the cycle which consists of only $E_{i}$-edges for some $i$. A graph structure is a tree if it is connected and contains no cycle or equivalently the underlying graph is a tree. $G^{*}$ is an $E_{i}$-tree, if the subgraph structure induced by $E_{i}$-edges is a tree. Similarly, $G^{*}$ is an $E_{1} E_{2} \cdots E_{j}$-tree, if $G^{*}$ is an $E_{i}$-tree for each $j, 1 \leq j \leq k$. A graph structure is an $E_{i}$-forest, if the subgraph structure induced by $E_{i}$-edges is a forest, that is, if it has no $E_{i}$-cycles. Let $S \subseteq U$; then the subgraph structure $\langle S\rangle$ induced by $S$ has vertex set $S$, where two vertices $u$ and $v$ in $\langle S\rangle$ are joined by an $E_{i}$-edge, $1 \leq i \leq k$, if and only if, they are joined by an $E_{i}$-edge in $G^{*}$. For some $i, 1 \leq i \leq k$, the $E_{i}$-subgraph induced by $S$ is denoted by $E_{i}-\langle S\rangle$. It has only those $E_{i}$-edges of $G^{*}$, joining vertices in $S$. If $T$ is a subset of edge set in $G^{*}$, then subgraph structure $\langle T\rangle$ induced by $T$ has the vertex set, "the end vertices in $T$ ", whose edges are those in $T$. Let $G^{*}=\left(U_{1}, E_{1}, E_{2}, \ldots, E_{m}\right)$ and $H^{*}=\left(U_{2}, E_{1}^{\prime}, E_{2}^{\prime}, \ldots, E_{n}^{\prime}\right)$ be graph structures. Then $G^{*}$ and $H^{*}$ are isomorphic, if (i) $m=n$, (ii) there exist a bijection $f: U_{1} \rightarrow U_{2}$ and a bijection $\phi:\left\{E_{1}, E_{2}, \ldots, E_{n}\right\} \rightarrow\left\{E_{1}^{\prime}, E_{2}^{\prime}, \ldots, E_{n}^{\prime}\right\}$, say $E_{i} \rightarrow E_{j}^{\prime}$, $1 \leq i, j \leq n$, such that for all $u, v \in U_{1}, u v \in E_{i}$ implies that $f(u) f(v) \in E_{j}^{\prime}$.

Two graph structures $G^{*}=\left(U, E_{1}, E_{2}, \ldots, E_{k}\right)$ and $H^{*}=$ $\left(U, E_{1}^{\prime}, E_{2}^{\prime}, \ldots, E_{k}^{\prime}\right)$, on the same vertex set $U$, are identical, if there exists a bijection $f: U \rightarrow U$, such that for all $u$ and $v$ in $U, u v$ is an $E_{i}$-edge in $G^{*}$, then $f(u) f(v)$ is an $E_{i}^{\prime}$-edge in $H^{*}$, where $1 \leq i \leq k$ and $E_{i} \simeq E_{i}^{\prime} \forall i$. Let $\phi$ be a permutation on $\left\{E_{1}, E_{2}, \ldots, E_{k}\right\}$. Then the $\phi$-cyclic complement of $G^{*}$, denoted by $\left(G^{*}\right)^{\phi c}$, is obtained by replacing $E_{i}$ by $\phi\left(E_{i}\right), 1 \leq i \leq k$. Let $G^{*}=\left(U, E_{1}, E_{2}, \ldots, E_{k}\right)$ be a graph structure and $\phi$ a permutation on $\left\{E_{1}, E_{2}, \ldots, E_{k}\right\}$; then

(i) $G^{*}$ is $\phi$-self complementary, if $G^{*}$ is isomorphic to $\left(G^{*}\right)^{\phi c}$; the $\phi$-cyclic complement of $G^{*}$ and $G^{*}$ is selfcomplement, if $\phi \neq$ identity permutation.

(ii) $G^{*}$ is strong $\phi$-self complementary, if $G^{*}$ is identical to $\left(G^{*}\right)^{\phi c}$; the $\phi$-complement of $G^{*}$ and $G^{*}$ is strong selfcomplement, if $\phi \neq$ identity permutation.

Definition 1 (see [2]). A fuzzy subset $\mu$ on a set $X$ is a map $\mu: X \rightarrow[0,1]$. A fuzzy binary relation on $X$ is a fuzzy subset $\mu$ on $X \times X$. By a fuzzy relation we mean a fuzzy binary relation given by $\mu: X \times X \rightarrow[0,1]$.

Definition 2 (see [8]). Let $G^{*}=\left(U, E_{1}, E_{2}, \ldots, E_{k}\right)$ be a graph structure and let $\nu, \rho_{1}, \rho_{2}, \ldots, \rho_{k}$ be the fuzzy subsets of $U, E_{1}, E_{2}, \ldots, E_{k}$, respectively, such that

$$
0 \leq \rho_{i}(x y) \leq \mu(x) \wedge \mu(y)
$$

$$
\forall x, y \in U, i=1,2, \ldots, k \text {. }
$$

Then $G=\left(\nu, \rho_{1}, \rho_{2}, \ldots, \rho_{k}\right)$ is a fuzzy graph structure of $G^{*}$.
Definition 3 (see [8]). Let $G=\left(\nu, \rho_{1}, \rho_{2}, \ldots, \rho_{k}\right)$ be a fuzzy graph structure of a graph structure $G^{*}=\left(U, E_{1}, E_{2}, \ldots, E_{k}\right)$. Then $F=\left(\nu, \tau_{1}, \tau_{2}, \ldots, \tau_{k}\right)$ is a partial fuzzy spanning subgraph structure of $G$ if $\tau_{i} \subseteq \rho_{i}$ for $i=1,2, \ldots, k$.

Definition 4 (see [8]). Let $G^{*}$ be a graph structure and let $G$ be a fuzzy graph structure of $G^{*}$. If $x y \in \operatorname{supp}\left(\rho_{i}\right)$, then " $x y$ " is said to be a $\rho_{i}$-edge of $G$.

Definition 5 (see [8]). The strength of a $\rho_{i}$-path $x_{0} x_{1} \cdots x_{n}$ of a fuzzy graph structure $G$ is $\bigwedge_{j=1}^{n} \rho_{i}\left(x_{j-1} x_{j}\right)$ for $i=1,2, \ldots, k$.

Definition 6 (see [8]). In a fuzzy graph structure $G, \rho_{i}^{2}(x y)=$ $\rho_{i} \circ \rho_{i}(x y)=\bigvee_{z}\left\{\rho_{i}(x z) \wedge \rho_{i}(z y)\right\}, \rho_{i}^{j}(x y)=\left(\rho_{i}^{j-1} \circ \rho_{i}\right)(x y)=$ $\bigvee_{z}\left\{\rho_{i}^{j-1}(x z) \wedge \rho_{i}(z y)\right\}, j=2,3, \ldots, m$, for any $m \geq 2$. Also $\rho_{i}^{\infty}(x y)=\bigvee\left\{\rho_{i}^{j}(x y), j=1,2, \ldots\right\}$.

Definition 7 (see [8]). Let $x y$ be a $\rho_{i}$-edge of $G=$ $\left(\nu, \rho_{1}, \rho_{2}, \ldots, \rho_{n}\right)$. Let $\left(\nu, \rho_{1}^{\prime}, \rho_{2}^{\prime}, \ldots, \rho_{n}^{\prime}\right)$ be a partial fuzzy spanning subgraph structure obtained by deleting " $x y$ " with $\rho_{i}^{\prime}(x y)=0$ and $\rho_{i}^{\prime}\left(x_{1} y_{1}\right)=\rho_{i}\left(x_{1} y_{1}\right) \forall \rho_{i}$-edges $\left(x_{1} y_{1}\right)$ other than $(x, y)$. If $\rho_{i}^{\infty}(u v)>\rho_{i}^{\prime \infty}(u v)$ for some $u v \in \operatorname{supp}\left(\rho_{i}\right)$, then $x y$ is a $\rho_{i}$-bridge.

Definition 8 (see [8]). Let $G^{\prime}=\left(\nu, \rho_{1}^{\prime}, \rho_{2}^{\prime}, \ldots, \rho_{n}^{\prime}\right)$ be the partial fuzzy subgraph structure obtained by deleting vertex $w$ of $G$, that is, $\nu^{\prime}(w)=0$ and $\nu^{\prime}(v)=v(v) \forall v \neq w, \quad \rho_{i}^{\prime}(v w)=0 \forall v \in$ $U r$ and $\rho_{i}^{\prime}(u v)=\rho_{i}(u v) \forall u v \neq w v, i=1,2, \ldots, k$. Then a vertex $w$ of $G$ is a $\rho_{i}$-cut vertex if $\rho_{i}^{\infty}(u v)>\rho_{i}^{\prime \infty}(u v)$ for some $u, v$ with $u, v \neq w$.

Definition 9 (see [8]). $G=\left(\nu, \rho_{1}, \rho_{2}, \ldots, \rho_{k}\right)$ is a $\rho_{i}$-cycle if and only if $\left(\operatorname{supp}(\nu), \operatorname{supp}\left(\rho_{1}\right), \operatorname{supp}\left(\rho_{2}\right), \ldots, \operatorname{supp}\left(\rho_{k}\right)\right)$ is a $E_{i^{-}}$ cycle.

Definition 10 (see [8]). $G=\left(\nu, \rho_{1}, \rho_{2}, \ldots, \rho_{k}\right)$ is a fuzzy $\rho_{i}$ cycle if and only if $\left(\operatorname{supp}(\nu), \operatorname{supp}\left(\rho_{1}\right), \operatorname{supp}\left(\rho_{2}\right), \ldots, \operatorname{supp}\left(\rho_{k}\right)\right)$ is an $E_{i}$-cycle and there exists no unique " $x y$ " in $\operatorname{supp}\left(\rho_{i}\right)$ such that $\rho_{i}(x y)=\bigwedge\left\{\rho_{i}(u v) \mid u v \in \operatorname{supp}\left(\rho_{i}\right)\right\}$.

Definition 11 (see [8]). $G=\left(\nu, \rho_{1}, \rho_{2}, \ldots, \rho_{k}\right)$ is a fuzzy $\rho_{i}$ tree if it has a partial fuzzy spanning subgraph structure, $F_{i}=\left(\nu, \tau_{1}, \tau_{2}, \ldots, \tau_{k}\right)$, which is a $\tau_{i}$-tree where for all $\rho_{i}$-edges not in $F_{i}, \rho_{i}(x y)<\tau_{i}^{\infty}(x y)$.

Definition 12 (see [8]). Let $G^{*}=\left(U, E_{1}, E_{2}, \ldots, E_{k}\right)$ be a graph structure and let $\nu, \rho_{1}, \rho_{2}, \ldots, \rho_{k}$ be the fuzzy subsets of $U, E_{1}, E_{2}, \ldots, E_{k}$, respectively, such that

$$
0 \leq \rho_{i}(x y) \leq \mu(x) \wedge \mu(y) \quad \forall x, y \in V, i=1,2, \ldots, k .
$$

Then $G=\left(\nu, \rho_{1}, \rho_{2}, \ldots, \rho_{k}\right)$ is a fuzzy graph structure of $G^{*}$.

Definition 13 (see [3]). Let $X$ be a nonempty set. A bipolar fuzzy set $B$ in $X$ is an object having the form

$$
B=\left\{\left(x, \mu_{B}^{P}(x), \mu_{B}^{N}(x)\right) \mid x \in X\right\},
$$

where $\mu_{B}^{P}: X \rightarrow[0,1]$ and $\mu_{B}^{N}: X \rightarrow[-1,0]$ are mappings. 
We use the positive membership degree $\mu_{B}^{P}(x)$ to denote the satisfaction degree of an element $x$ to the property corresponding to a bipolar fuzzy set $B$ and the negative membership degree $\mu_{B}^{N}(x)$ to denote the satisfaction degree of an element $x$ to some implicit counterproperty corresponding to a bipolar fuzzy set $B$. If $\mu_{B}^{P}(x) \neq 0$ and $\mu_{B}^{N}(x)=0$, it is the situation that $x$ is regarded as having only positive satisfaction for $B$. If $\mu_{B}^{P}(x)=0$ and $\mu_{B}^{N}(x) \neq 0$, it is the situation that $x$ does not satisfy the property of $B$ but somewhat satisfies the counter property of $B$. It is possible for an element $x$ to be such that $\mu_{B}^{P}(x) \neq 0$ and $\mu_{B}^{N}(x) \neq 0$ when the membership function of the property overlaps that of its counterproperty over some portion of $X$.

For the sake of simplicity, we will use the symbol $B=$ $\left(\mu_{B}^{P}, \mu_{B}^{N}\right)$ for the bipolar fuzzy set:

$$
B=\left\{\left(x, \mu_{B}^{P}(x), \mu_{B}^{N}(x)\right) \mid x \in X\right\} .
$$

Definition 14 (see [3]). Let $X$ be a nonempty set. Then we call a mapping $A=\left(\mu_{A}^{P}, \mu_{A}^{N}\right): X \times X \rightarrow[0,1] \times[-1,0]$ a bipolar fuzzy relation on $X$ such that $\mu_{A}^{P}(x, y) \in[0,1]$ and $\mu_{A}^{N}(x, y) \in$ $[-1,0]$.

Definition 15 (see [9]). A bipolar fuzzy graph $G=(V, A, B)$ is a nonempty set $V$ together with a pair of functions $A=$ $\left(\mu_{A}^{P}, \mu_{A}^{N}\right): V \rightarrow[0,1] \times[-1,0]$ and $B=\left(\mu_{B}^{P}, \mu_{B}^{N}\right): V \times V \rightarrow$ $[0,1] \times[-1,0]$ such that for all $x, y \in V$,

$$
\begin{aligned}
& \mu_{B}^{P}(x, y) \leq \min \left(\mu_{A}^{P}(x), \mu_{A}^{P}(y)\right), \\
& \mu_{B}^{N}(x, y) \geq \max \left(\mu_{A}^{N}(x), \mu_{A}^{N}(y)\right) .
\end{aligned}
$$

Notice that $\mu_{B}^{P}(x, y)>0, \mu_{B}^{N}(x, y)<0$ for $(x, y) \in V \times V$, $\mu_{B}^{P}(x, y)=\mu_{B}^{N}(x, y)=0$ for $(x, y) \notin V \times V$, and $B$ is symmetric relation.

\section{Bipolar Fuzzy Graph Structures}

Definition 16. $\check{G}_{b}=\left(M, N_{1}, N_{2}, \ldots, N_{n}\right)$ is called a bipolar fuzzy graph structure (BFGS) of a graph structure (GS) $G^{*}=$ $\left(U, E_{1}, E_{2}, \ldots, E_{n}\right)$ if $M=\left(\mu_{M}^{P}, \mu_{M}^{N}\right)$ is a bipolar fuzzy set on $U$ and for each $i=1,2, \ldots, n ; N_{i}=\left(\mu_{N_{i}}^{P}, \mu_{N_{i}}^{N}\right)$ is a bipolar fuzzy set on $E_{i}$ such that

$$
\begin{aligned}
& \mu_{N_{i}}^{P}(x y) \leq \mu_{M}^{P}(x) \wedge \mu_{M}^{P}(y), \\
& \mu_{N_{i}}^{N}(x y) \geq \mu_{M}^{N}(x) \vee \mu_{M}^{N}(y)
\end{aligned}
$$

$\forall x y \in E_{i} \subset U \times U$.

Note that $\mu_{N_{i}}^{P}(x y)=0=\mu_{N_{i}}^{N}(x y)$ for all $x y \in U \times U-E_{i}$ and $0<\mu_{N_{i}}^{P}(x y) \leq 1,-1 \leq \mu_{N_{i}}^{N}(x y)<0 \forall x y \in E_{i}$, where $U$ and $E_{i}(i=1,2, \ldots, n)$ are called underlying vertex set and underlying $i$-edge set of $\check{G}_{b}$, respectively.

Definition 17. Let $\check{G}_{b}=\left(M, N_{1}, N_{2}, \ldots, N_{n}\right)$ be a bipolar fuzzy graph structure of a graph structure $G^{*}=\left(U, E_{1}, E_{2}, \ldots, E_{n}\right)$.
If $\check{H}_{b}=\left(M^{\prime}, N_{1}^{\prime}, N_{2}^{\prime}, \ldots, N_{n}^{\prime}\right)$ is a bipolar fuzzy graph structure of $G^{*}$ such that

$$
\begin{aligned}
& \mu_{M^{\prime}}^{P}(x) \leq \mu_{M}^{P}(x), \\
& \mu_{M^{\prime}}^{N}(x) \geq \mu_{M}^{N}(x) \quad \forall x \in U, \\
& \mu_{N_{i}^{\prime}}^{P}(x y) \leq \mu_{N_{i}}^{P}(x y), \\
& \mu_{N_{i}^{\prime}}^{N}(x y) \geq \mu_{N_{i}}^{N}(x y)
\end{aligned}
$$

$\forall x y \in E_{i}, i=1,2, \ldots, n$,

then $\check{H}_{b}$ is called a bipolar fuzzy subgraph structure of BFGS $\check{G}_{b}$.

BFGS $\check{H}_{b}=\left(M^{\prime}, N_{1}^{\prime}, N_{2}^{\prime}, \ldots, N_{n}^{\prime}\right)$ is a bipolar fuzzy induced subgraph structure of $\check{G}_{b}=\left(M, N_{1}, N_{2}, \ldots, N_{n}\right)$, by a subset $W$ of $U$ if

$$
\begin{aligned}
& \mu_{M^{\prime}}^{P}(x)=\mu_{M}^{P}(x), \\
& \mu_{M^{\prime}}^{N}(x)=\mu_{M}^{N}(x)
\end{aligned}
$$

$\forall x \in W$,

$$
\begin{aligned}
& \mu_{N_{i}^{\prime}}^{P}(x y)=\mu_{N_{i}}^{P}(x y), \\
& \mu_{N_{i}^{\prime}}^{N}(x y)=\mu_{N_{i}}^{N}(x y)
\end{aligned}
$$

$$
\forall x, y \in W, i=1,2, \ldots, n .
$$

Similarly, BFGS $\check{H}_{b}$ is a bipolar fuzzy spanning subgraph structure of $\check{G}_{b}$ if $M^{\prime}=M$ and

$$
\begin{aligned}
& \mu_{N_{i}^{\prime}}^{P} \leq \mu_{N_{i}}^{P}, \\
& \mu_{N_{i}^{\prime}}^{N} \geq \mu_{N_{i}}^{N},
\end{aligned}
$$

$$
i=1,2, \ldots, n .
$$

Example 18. Consider a graph structure $G^{*}=\left(U, E_{1}, E_{2}\right)$ such that $U=\left\{a_{1}, a_{2}, a_{3}, a_{4}\right\}, E_{1}=\left\{a_{1} a_{2}, a_{2} a_{4}\right\}$, and $E_{2}=$ $\left\{a_{3} a_{4}, a_{1} a_{4}\right\}$.

(i) Let $M, N_{1}$, and $N_{2}$ be bipolar fuzzy subsets of $U, E_{1}$, and $E_{2}$, respectively, such that

$$
\begin{aligned}
& M=\left\{\left(a_{1}, 0.5,-0.2\right),\left(a_{2}, 0.7,-0.3\right),\left(a_{3}, 0.4,-0.3\right),\right. \\
& \left.\quad\left(a_{4}, 0.7,-0.3\right)\right\}, \\
& N_{1}=\left\{\left(a_{1} a_{2}, 0.5,-0.2\right),\left(a_{2} a_{4}, 0.7,-0.3\right)\right\}, \\
& N_{2}=\left\{\left(a_{3} a_{4}, 0.3,-0.2\right),\left(a_{1} a_{4}, 0.3,-0.1\right)\right\} .
\end{aligned}
$$

Then, by direct calculations, it is easy to see that $\check{G}_{b}=$ $\left(M, N_{1}, N_{2}\right)$ is a BFGS of $G^{*}$ as shown in Figure 1.

(ii) Consider $M_{1}=\left\{\left(a_{1}, 0.4,-0.1\right),\left(a_{2}, 0.5,-0.3\right),\left(a_{3}\right.\right.$, $\left.0.4,-0.2),\left(a_{4}, 0.1,-0.3\right)\right\}, N_{11}=\left\{\left(a_{1} a_{2}, 0.4,-0.1\right),\left(a_{2} a_{4}, 0.1\right.\right.$, $-0.2)\}$, and $N_{12}=\left\{\left(a_{3} a_{4}, 0.1,-0.2\right),\left(a_{1} a_{4}, 0.1,-0.0\right)\right\}$. Then, 


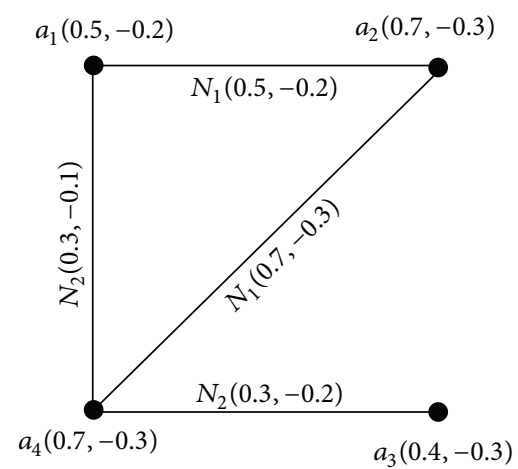

Figure 1: $\check{G}_{b}=\left(M, N_{1}, N_{2}\right)$.

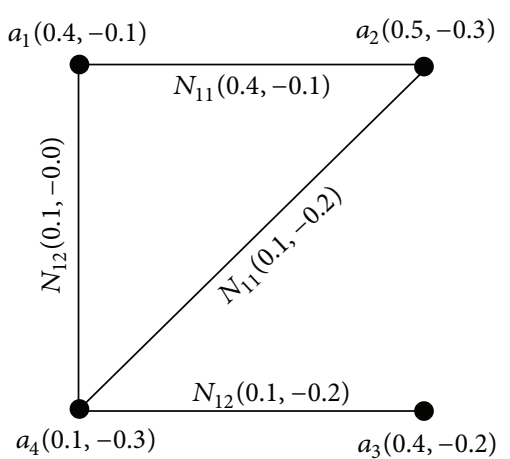

FIGURE 2: Bipolar fuzzy subgraph structure $\check{K}_{b}=\left(M_{1}, N_{11}, N_{12}\right)$.

by routine calculations, it is easy to see that $\check{K}_{b}=$ $\left(M_{1}, N_{11}, N_{12}\right)$ is the bipolar fuzzy subgraph structure of $\check{G}_{b}$ as shown in Figure 2.

Definition 19. Let $\check{G}_{b}=\left(M, N_{1}, N_{2}, \ldots, N_{n}\right)$ be a bipolar fuzzy graph structure of a graph structure $G^{*}=\left(U, E_{1}, E_{2}, \ldots, E_{n}\right)$. Then $x y \in E_{i}$ is called a bipolar fuzzy $N_{i}$-edge or simply $N_{i^{-}}$ edge, if

$$
\begin{aligned}
& \mu_{N_{i}}^{P}(x y)>0 \text { or } \\
& \mu_{N_{i}}^{N}(x y)<0 .
\end{aligned}
$$

Then support of $N_{i}, i=1,2, \ldots, n$, consequently, is

$$
\operatorname{supp}\left(N_{i}\right)=\left\{x y \in E_{i}: \mu_{N_{i}}^{P}(x y)>0, \mu_{N_{i}}^{N}(x y)<0\right\} .
$$

Definition 20. $N_{i}$-path in a BFGS $\check{G}_{b}=\left(M, N_{1}, N_{2}, \ldots, N_{n}\right)$ of a graph structure $G^{*}=\left(U, E_{1}, E_{2}, \ldots, E_{n}\right)$ is a sequence $a_{1}, a_{2}, \ldots, a_{m}$ of distinct vertices (except the choice $a_{m}=a_{1}$ ) in $U$, such that $a_{j-1} a_{j}$ is a bipolar fuzzy $N_{i}$-edge for all $j=$ $2,3, \ldots, m$.

Definition 21. A BFGS $\check{G}_{b}=\left(M, N_{1}, N_{2}, \ldots, N_{n}\right)$ with underlying vertex set $U$ is said to be $N_{i}$-strong for some $i \in$ $\{1,2,3, \ldots, n\}$ if for all $x y \in \operatorname{supp}\left(N_{i}\right)$

$$
\begin{aligned}
& \mu_{N_{i}}^{P}(x y)=\mu_{M}^{P}(x) \wedge \mu_{M}^{P}(y), \\
& \mu_{N_{i}}^{N}(x y)=\mu_{M}^{N}(x) \vee \mu_{M}^{N}(y) .
\end{aligned}
$$

A BFGS $\check{G}_{b}=\left(M, N_{1}, N_{2}, \ldots, N_{n}\right)$ is said to be strong if it is $N_{i}$-strong BFGS for all $i \in\{1,2,3, \ldots, n\}$.

Example 22. Consider BFGS $\check{G}_{b}=\left(M, N_{1}, N_{2}\right)$ as shown in Figure 3. strong.

Then $\check{G}_{b}$ is a strong BFGS since it is both $N_{1}$ - and $N_{2}$ -

Definition 23. A BFGS $\check{G}_{b}=\left(M, N_{1}, N_{2}, \ldots, N_{n}\right)$ with underlying vertex set $U$ is said to be complete or $N_{1} N_{2} \cdots N_{n^{-}}$complete, if the following are true:

(i) $\check{G}_{b}$ a is strong BFGS.

(ii) $\operatorname{supp}\left(N_{i}\right) \neq \emptyset \forall i=1,2,3, \ldots, n$.

(iii) For each pair of vertices $x, y \in U, x y$ is an $N_{i}$-edge for some $i$.

Example 24. Let $\check{G}_{b}=\left(M, N_{1}, N_{2}\right)$ be BFGS of graph structure $G^{*}=\left(U, E_{1}, E_{2}\right)$ such that $U=\left\{a_{1}, a_{2}, a_{3}\right\}, E_{1}=$ $\left\{a_{2} a_{3}\right\}$, and $E_{2}=\left\{a_{1} a_{2}, a_{1} a_{3}\right\}$ as shown in Figure 4 . By routine calculations, it is easy to see that $\check{G}_{b}$ is a strong BFGS.

Moreover, $\operatorname{supp}\left(N_{1}\right) \neq \emptyset$, $\operatorname{supp}\left(N_{2}\right) \neq \emptyset$, and every pair of vertices belonging to $U$ is either an $N_{1}$-edge or an $N_{2}$-edge. So $\check{G}_{b}$ is a complete BFGS, that is, $N_{1} N_{2}$-complete BFGS.

Definition 25. Let $\check{G}_{b}=\left(M, N_{1}, N_{2}, \ldots, N_{n}\right)$ be a BFGS with underlying vertex set $U$. Then positive and negative strengths of a $N_{i}$-path " $P_{N_{i}}=a_{1} a_{2} \cdots a_{m}$ " are called gain and loss of that $N_{i}$-path and denoted by G.P. $P_{N_{i}}$ and $L . P_{N_{i}}$, respectively, such that

$$
\begin{aligned}
& \text { G.P } P_{N_{i}}=\bigwedge_{j=2}^{m}\left[\mu_{N_{i}}^{P}\left(a_{j-1} a_{j}\right)\right], \\
& L . P_{N_{i}}=\left|\bigvee_{j=2}^{m} \mu_{N_{i}}^{N}\left(a_{j-1} a_{j}\right)\right| .
\end{aligned}
$$

Example 26. Consider a BFGS $\check{G}_{b}=\left(M, N_{1}, N_{2}\right)$ as shown in Figure 4. We note that $P_{N_{2}}=a_{1} a_{3} a_{4} a_{1}$ is an $N_{2}$-path. So G. $P_{N_{2}}=\mu_{N_{2}}^{P}\left(a_{3} a_{1}\right) \wedge \mu_{N_{2}}^{P}\left(a_{1} a_{2}\right)=0.5 \wedge 0.4=0.4$. Consider

$$
\begin{aligned}
L . P_{N_{2}} & =\left|\mu_{N_{2}}^{N}\left(a_{3} a_{1}\right) \vee \mu_{N_{2}}^{N}\left(a_{1} a_{2}\right)\right|=|-0.4 \vee-0.4| \\
& =|-0.4|=0.4 .
\end{aligned}
$$

Definition 27. Let $\check{G}_{b}=\left(M, N_{1}, N_{2}, \ldots, N_{n}\right)$ be a BFGS with underlying vertex set $U$. Then

(i) $N_{i}$-gain of connectedness between $x$ and $y$ is defined by $\mu_{N_{i}}^{\infty,+}(x y)=\bigvee_{j \geq 1}\left\{\mu_{N_{i}}^{j,+}(x y)\right\}$, such that $\mu_{N_{i}}^{j,+}(x y)=$ $\left(\mu_{N_{i}}^{j-1,+} \circ \mu_{N_{i}}^{1,+}\right)(x y)$ for $j \geq 2$ and $\mu_{N_{i}}^{2,+}(x y)=\left(\mu_{N_{i}}^{1,+}\right.$ 。 $\left.\mu_{N_{i}}^{1,+}\right)(x y)=\bigvee_{z}\left\{\mu_{N_{i}}^{1,+}(x z) \wedge \mu_{N_{i}}^{1,+}(z y)\right\}$, where $\mu_{N_{i}}^{1,+}=$ $\mu_{N_{i}}^{P}, \forall i$.

(ii) $N_{i}$-loss of connectedness between $x$ and $y$ is defined by $\mu_{N_{i}}^{\infty,-}(x y)=\bigvee_{j \geq 1}\left\{\mu_{N_{i}}^{j,-}(x y)\right\}$, such that $\mu_{N_{i}}^{j,-}(x y)=$ $\left(\mu_{N_{i}}^{j-1,-} \circ \mu_{N_{i}}^{1,-}\right)(x y)$ for $j \geq 2$ and $\mu_{N_{i}}^{2,-}(x y)=\left(\mu_{N_{i}}^{1,-}\right.$ 。 


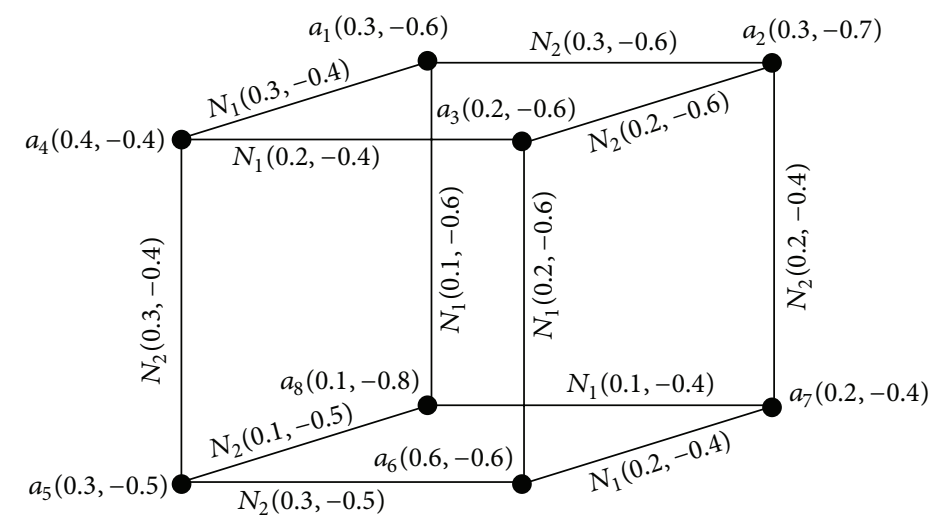

Figure 3: BFGS $\check{G}_{b}=\left(M, N_{1}, N_{2}\right)$.

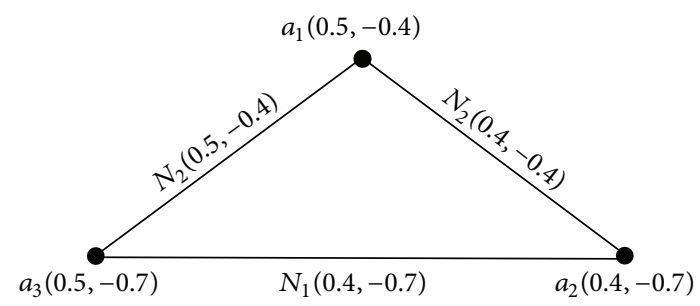

FIgURE 4: $\check{G}_{b}=\left(M, N_{1}, N_{2}\right)$.

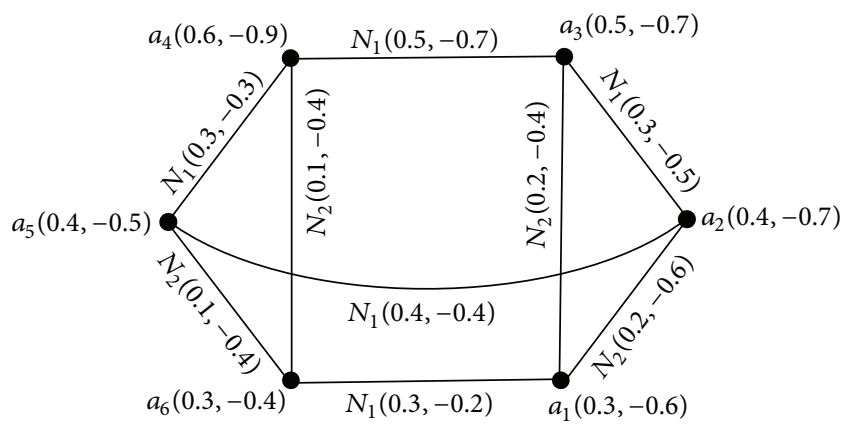

Figure 5: $\check{G}_{b}=\left(M, N_{1}, N_{2}\right)$.

$\left.\mu_{N_{i}}^{1,-}\right)(x y)=\bigvee_{z}\left\{\mu_{N_{i}}^{1,-}(x z) \wedge \mu_{N_{i}}^{1,-}(z y)\right\}$, where $\mu_{N_{i}}^{1,-}=$ $\left|\mu_{N_{i}}^{N}\right|, \forall i$.

Example 28. Let $\check{G}_{b}=\left(M, N_{1}, N_{2}\right)$ be BFGS of graph structure $G=\left(U, E_{1}, E_{2}\right)$ such that $U=\left\{a_{1}, a_{2}, a_{3}, a_{4}, a_{5}, a_{6}\right\}, E_{1}=$ $\left\{a_{1} a_{6}, a_{2} a_{3}, a_{2} a_{5}, a_{3} a_{4}, a_{4} a_{5}\right\}$, and $E_{2}=\left\{a_{1} a_{3}, a_{1} a_{2}, a_{4} a_{6}, a_{5} a_{6}\right\}$, as is shown in Figure 5.

Since $\mu_{N_{1}}^{1,+}\left(a_{2} a_{3}\right)=0.3, \mu_{N_{1}}^{1,+}\left(a_{2} a_{4}\right)=0.0, \mu_{N_{1}}^{1,+}\left(a_{2} a_{5}\right)=0.4$, $\mu_{N_{1}}^{1,+}\left(a_{3} a_{4}\right)=0.5, \mu_{N_{1}}^{1,+}\left(a_{5} a_{3}\right)=0.0, \mu_{N_{1}}^{1,+}\left(a_{4} a_{5}\right)=0.3$, and $\mu_{N_{1}}^{1,+}\left(a_{1} a_{6}\right)=0.3$, therefore

$$
\begin{aligned}
\mu_{N_{1}}^{2,+}\left(a_{2} a_{3}\right) & =\left(\mu^{1,+} n_{N_{1}} \circ \mu_{N_{1}}^{1,+}\right)\left(a_{2} a_{3}\right) \\
& =\left[\mu_{N_{1}}^{1,+}\left(a_{2} a_{4}\right) \wedge \mu_{N_{1}}^{1,+}\left(a_{4} a_{3}\right)\right]
\end{aligned}
$$

$$
\begin{aligned}
& \vee\left[\mu_{N_{1}}^{1,+}\left(a_{2} a_{5}\right) \wedge \mu_{N_{1}}^{1,+}\left(a_{5} a_{3}\right)\right] \\
= & {[0.0 \wedge 0.5] \vee[0.4 \wedge 0.0]=0, } \\
\mu_{N_{1}}^{2,+}\left(a_{2} a_{4}\right)= & \left(\mu^{1,+} n_{N_{1}} \circ \mu_{N_{1}}^{1,+}\right)\left(a_{2} a_{4}\right) \\
= & {\left[\mu_{N_{1}}^{2,+}\left(a_{2} a_{3}\right) \wedge \mu_{N_{1}}^{1,+}\left(a_{3} a_{4}\right)\right] } \\
& \vee\left[\mu_{N_{1}}^{1,+}\left(a_{2} a_{5}\right) \wedge \mu_{N_{1}}^{1,+}\left(a_{5} a_{4}\right)\right] \\
= & {[0.3 \wedge 0.5] \vee[0.4 \wedge 0.3]=0.3, } \\
\mu_{N_{1}}^{2,+}\left(a_{2} a_{5}\right)= & \left(\mu^{1,+} n_{N_{1}} \circ \mu_{N_{1}}^{1,+}\right)\left(a_{2} a_{5}\right) \\
= & {\left[\mu_{N_{1}}^{1,+}\left(a_{2} a_{3}\right) \wedge \mu_{N_{1}}^{1,+}\left(a_{3} a_{5}\right)\right] } \\
& \vee\left[\mu_{N_{1}}^{1,+}\left(a_{2} a_{4}\right) \wedge \mu_{N_{1}}^{1,+}\left(a_{4} a_{5}\right)\right] \\
= & {[0.3 \wedge 0.0] \vee[0.0 \wedge 0.3]=0, } \\
\mu_{N_{1}}^{2,+}\left(a_{3} a_{4}\right)= & \left(\mu^{1,+} n_{N_{1}} \circ \mu_{N_{1}}^{1,+}\right)\left(a_{3} a_{4}\right) \\
= & {\left[\mu_{N_{1}}^{1,+}\left(a_{3} a_{2}\right) \wedge \mu_{N_{1}}^{1,+}\left(a_{2} a_{4}\right)\right] } \\
& \vee\left[\mu_{N_{1}}^{1,+}\left(a_{3} a_{5}\right) \wedge \mu_{N_{1}}^{1,+}\left(a_{5} a_{4}\right)\right] \\
= & {[0.3 \wedge 0.0] \vee[0.0 \wedge 0.3]=0, } \\
\mu_{N_{1}}^{2,+}\left(a_{4} a_{5}\right)= & \left(\mu^{1,+} n_{N_{1}} \circ \mu_{N_{1}}^{1,+}\right)\left(a_{4} a_{5}\right) \\
= & {\left[\mu_{N_{1}}^{1,+}\left(a_{4} a_{2}\right) \wedge \mu_{N_{1}}^{1,+}\left(a_{2} a_{5}\right)\right] } \\
& \vee\left[\mu_{N_{1}}^{1,+}\left(a_{4} a_{3}\right) \wedge \mu_{N_{1}}^{1,+}\left(a_{3} a_{5}\right)\right] \\
= & {[0.0 \wedge 0.4] \vee[0.5 \wedge 0.0]=0, } \\
\mu_{N_{1}}^{2,+}\left(a_{3} a_{5}\right)= & \left(\mu^{1,+} n_{N_{1}} \circ \mu_{N_{1}}^{1,+}\right)\left(a_{3} a_{5}\right) \\
= & {\left[\mu_{N_{1}}^{1,+}\left(a_{3} a_{2}\right) \wedge \mu_{N_{1}}^{1,+}\left(a_{2} a_{5}\right)\right] } \\
& \vee\left[\mu_{N_{1}}^{1,+}\left(a_{3} a_{4}\right) \wedge \mu_{N_{1}}^{1,+}\left(a_{4} a_{5}\right)\right] \\
= & {[0.3 \wedge 0.4] \vee[0.5 \wedge 0.3]=0.3, } \\
&
\end{aligned}
$$


6

$$
\begin{array}{rlrl}
\mu_{N_{1}}^{2,+}\left(a_{1} a_{6}\right)= & \left(\mu^{1,+} n_{N_{1}} \circ \mu_{N_{1}}^{1,+}\right)\left(a_{1} a_{6}\right)=0, & \mu_{N_{1}}^{4,+}\left(a_{3} a_{4}\right)=\left(\mu^{1,+} n_{N_{1}} \circ \mu_{N_{1}}^{1,+}\right)\left(a_{3} a_{4}\right)=0, \\
\mu_{N_{1}}^{3,+}\left(a_{2} a_{3}\right)=\left(\mu^{2,+} n_{N_{1}} \circ \mu_{N_{1}}^{1,+}\right)\left(a_{2} a_{3}\right) & \mu_{N_{1}}^{4,+}\left(a_{3} a_{5}\right)=\left(\mu^{1,+} n_{N_{1}} \circ \mu_{N_{1}}^{1,+}\right)\left(a_{3} a_{5}\right)=0.3, \\
= & {\left[\mu_{N_{1}}^{2,+}\left(a_{2} a_{4}\right) \wedge \mu_{N_{1}}^{1,+}\left(a_{4} a_{3}\right)\right]} & \mu_{N_{1}}^{4,+}\left(a_{4} a_{5}\right)=\left(\mu^{1,+} n_{N_{1}} \circ \mu_{N_{1}}^{1,+}\right)\left(a_{4} a_{5}\right)=0, \\
& \vee\left[\mu_{N_{1}}^{2,+}\left(a_{2} a_{5}\right) \wedge \mu_{N_{1}}^{1,+}\left(a_{5} a_{3}\right)\right] & \mu_{N_{1}}^{4,+}\left(a_{1} a_{6}\right)=\left(\mu^{1,+} n_{N_{1}} \circ \mu_{N_{1}}^{1,+}\right)\left(a_{1} a_{6}\right)=0 . \\
= & {[0.3 \wedge 0.5] \vee[0.0 \wedge 0.0]=0.3,} &
\end{array}
$$$$
\mu_{N_{1}}^{3,+}\left(a_{2} a_{4}\right)=\left(\mu^{2,+} n_{N_{1}} \circ \mu_{N_{1}}^{1,+}\right)\left(a_{2} a_{4}\right)
$$$$
=\left[\mu_{N_{1}}^{2,+}\left(a_{2} a_{3}\right) \wedge \mu_{N_{1}}^{1,+}\left(a_{3} a_{4}\right)\right]
$$$$
\vee\left[\mu_{N_{1}}^{2,+}\left(a_{2} a_{5}\right) \wedge \mu_{N_{1}}^{1,+}\left(a_{5} a_{4}\right)\right]
$$$$
=[0.0 \wedge 0.5] \vee[0.0 \wedge 0.3]=0.0,
$$$$
\mu_{N_{1}}^{3,+}\left(a_{2} a_{5}\right)=\left(\mu^{2,+} n_{N_{1}} \circ \mu_{N_{1}}^{1,+}\right)\left(a_{2} a_{5}\right)
$$$$
=\left[\mu_{N_{1}}^{2,+}\left(a_{2} a_{3}\right) \wedge \mu_{N_{1}}^{1,+}\left(a_{3} a_{5}\right)\right]
$$$$
\vee\left[\mu_{N_{1}}^{2,+}\left(a_{2} a_{4}\right) \wedge \mu_{N_{1}}^{1,+}\left(a_{4} a_{5}\right)\right]
$$$$
=[0.0 \wedge 0.0] \vee[0.3 \wedge 0.3]=0.3 \text {, }
$$$$
\mu_{N_{1}}^{3,+}\left(a_{3} a_{4}\right)=\left(\mu^{2,+} n_{N_{1}} \circ \mu_{N_{1}}^{1,+}\right)\left(a_{3} a_{4}\right)
$$$$
=\left[\mu_{N_{1}}^{2,+}\left(a_{3} a_{2}\right) \wedge \mu_{N_{1}}^{1,+}\left(a_{2} a_{4}\right)\right]
$$$$
\vee\left[\mu_{N_{1}}^{2,+}\left(a_{3} a_{5}\right) \wedge \mu_{N_{1}}^{1,+}\left(a_{5} a_{4}\right)\right]
$$$$
=[0.0 \wedge 0.0] \vee[0.3 \wedge 0.3]=0.3,
$$$$
\mu_{N_{1}}^{3,+}\left(a_{3} a_{5}\right)=\left(\mu^{2,+} n_{N_{1}} \circ \mu_{N_{1}}^{1,+}\right)\left(a_{3} a_{5}\right)
$$$$
=\left[\mu_{N_{1}}^{2,+}\left(a_{3} a_{2}\right) \wedge \mu_{N_{1}}^{1,+}\left(a_{2} a_{5}\right)\right]
$$$$
\vee\left[\mu_{N_{1}}^{2,+}\left(a_{3} a_{4}\right) \wedge \mu_{N_{1}}^{1,+}\left(a_{4} a_{5}\right)\right]
$$$$
=[0.0 \wedge 0.4] \vee[0.0 \wedge 0.3]=0.0,
$$$$
\mu_{N_{1}}^{3,+}\left(a_{4} a_{5}\right)=\left(\mu^{2,+} n_{N_{1}} \circ \mu_{N_{1}}^{1,+}\right)\left(a_{4} a_{5}\right)
$$$$
=\left[\mu_{N_{1}}^{2,+}\left(a_{4} a_{2}\right) \wedge \mu_{N_{1}}^{1,+}\left(a_{2} a_{5}\right)\right]
$$$$
\vee\left[\mu_{N_{1}}^{2,+}\left(a_{4} a_{3}\right) \wedge \mu_{N_{1}}^{1,+}\left(a_{3} a_{5}\right)\right]
$$$$
=[0.3 \wedge 0.4] \vee[0.0 \wedge 0.0]=0.3,
$$$$
\mu_{N_{1}}^{3,+}\left(a_{1} a_{6}\right)=\left(\mu^{2,+} n_{N_{1}} \circ \mu_{N_{1}}^{1,+}\right)\left(a_{1} a_{6}\right)=0 .
$$

(16)

Similarly,

$$
\begin{aligned}
& \mu_{N_{1}}^{4,+}\left(a_{2} a_{3}\right)=\left(\mu^{1,+} n_{N_{1}} \circ \mu_{N_{1}}^{1,+}\right)\left(a_{2} a_{3}\right)=0, \\
& \mu_{N_{1}}^{4,+}\left(a_{2} a_{4}\right)=\left(\mu^{1,+} n_{N_{1}} \circ \mu_{N_{1}}^{1,+}\right)\left(a_{2} a_{4}\right)=0.3, \\
& \mu_{N_{1}}^{4,+}\left(a_{2} a_{5}\right)=\left(\mu^{1,+} n_{N_{1}} \circ \mu_{N_{1}}^{1,+}\right)\left(a_{2} a_{5}\right)=0,
\end{aligned}
$$

Applied Computational Intelligence and Soft Computing

This implies that

$$
\begin{aligned}
& \mu_{N_{1}}^{\infty,+}\left(a_{2} a_{3}\right)=\vee\{0.3,0.0,0.3,0.0\}=0.3, \\
& \mu_{N_{1}}^{\infty,+}\left(a_{2} a_{4}\right)=\vee\{0.0,0.3,0.0,0.3\}=0.3, \\
& \mu_{N_{1}}^{\infty,+}\left(a_{2} a_{5}\right)=\vee\{0.4,0.0,0.3,0.0\}=0.4, \\
& \mu_{N_{1}}^{\infty,+}\left(a_{3} a_{4}\right)=\vee\{0.5,0.0,0.3,0.0\}=0.5, \\
& \mu_{N_{1}}^{\infty,+}\left(a_{3} a_{5}\right)=\vee\{0.0,0.3,0.0,0.3\}=0.3, \\
& \mu_{N_{1}}^{\infty,+}\left(a_{4} a_{5}\right)=\vee\{0.3,0.0,0.3,0.0\}=0.3, \\
& \mu_{N_{1}}^{\infty,+}\left(a_{1} a_{6}\right)=\vee\{0.3,0.0,0.0,0.0\}=0.3 .
\end{aligned}
$$

Since

$$
\begin{aligned}
& \mu_{N_{1}}^{1,-}\left(a_{2} a_{3}\right)=0.5, \\
& \mu_{N_{1}}^{1,-}\left(a_{2} a_{4}\right)=0.0, \\
& \mu_{N_{1}}^{1,-}\left(a_{2} a_{5}\right)=0.4, \\
& \mu_{N_{1}}^{1,-}\left(a_{3} a_{4}\right)=0.7, \\
& \mu_{N_{1}}^{1,-}\left(a_{5} a_{3}\right)=0.0, \\
& \mu_{N_{1}}^{1,-}\left(a_{4} a_{5}\right)=0.3, \\
& \mu_{N_{1}}^{1,-}\left(a_{1} a_{6}\right)=0.2,
\end{aligned}
$$

we have

$$
\begin{aligned}
\mu_{N_{1}}^{2,-}\left(a_{2} a_{3}\right)= & \left(\mu^{1,-} n_{N_{1}} \circ \mu_{N_{1}}^{1,-}\right)\left(a_{2} a_{3}\right) \\
= & {\left[\mu_{N_{1}}^{1,-}\left(a_{2} a_{4}\right) \wedge \mu_{N_{1}}^{1,-}\left(a_{4} a_{3}\right)\right] } \\
& \vee\left[\mu_{N_{1}}^{1,-}\left(a_{2} a_{5}\right) \wedge \mu_{N_{1}}^{1,-}\left(a_{5} a_{3}\right)\right] \\
= & {[0.0 \wedge 0.7] \vee[0.4 \wedge 0.0]=0.0, }
\end{aligned}
$$

$$
\begin{aligned}
\mu_{N_{1}}^{2,-}\left(a_{2} a_{4}\right)= & \left(\mu^{1,-} n_{N_{1}} \circ \mu_{N_{1}}^{1,-}\right)\left(a_{2} a_{4}\right) \\
= & {\left[\mu_{N_{1}}^{2,-}\left(a_{2} a_{3}\right) \wedge \mu_{N_{1}}^{1,-}\left(a_{3} a_{4}\right)\right] } \\
& \vee\left[\mu_{N_{1}}^{1,-}\left(a_{2} a_{5}\right) \wedge \mu_{N_{1}}^{1,-}\left(a_{5} a_{4}\right)\right] \\
= & {[0.5 \wedge 0.7] \vee[0.4 \wedge 0.3]=0.5, }
\end{aligned}
$$


Applied Computational Intelligence and Soft Computing

7

$$
\begin{aligned}
& \mu_{N_{1}}^{2,-}\left(a_{2} a_{5}\right)=\left(\mu^{1,-} n_{N_{1}} \circ \mu_{N_{1}}^{1,-}\right)\left(a_{2} a_{5}\right) \\
& =\left[\mu_{N_{1}}^{1,-}\left(a_{2} a_{3}\right) \wedge \mu_{N_{1}}^{1,-}\left(a_{3} a_{5}\right)\right] \\
& \vee\left[\mu_{N_{1}}^{1,-}\left(a_{2} a_{4}\right) \wedge \mu_{N_{1}}^{1,-}\left(a_{4} a_{5}\right)\right] \\
& =[0.5 \wedge 0.0] \vee[0.0 \wedge 0.3]=0.0, \\
& \mu_{N_{1}}^{2,-}\left(a_{3} a_{4}\right)=\left(\mu^{1,-} n_{N_{1}} \circ \mu_{N_{1}}^{1,-}\right)\left(a_{3} a_{4}\right) \\
& =\left[\mu_{N_{1}}^{1,-}\left(a_{3} a_{2}\right) \wedge \mu_{N_{1}}^{1,-}\left(a_{2} a_{4}\right)\right] \\
& \vee\left[\mu_{N_{1}}^{1,-}\left(a_{3} a_{5}\right) \wedge \mu_{N_{1}}^{1,-}\left(a_{5} a_{4}\right)\right] \\
& =[0.5 \wedge 0.0] \vee[0.0 \wedge 0.3]=0.0, \\
& \mu_{N_{1}}^{2,-}\left(a_{3} a_{5}\right)=\left(\mu^{1,-} n_{N_{1}} \circ \mu_{N_{1}}^{1,-}\right)\left(a_{3} a_{5}\right) \\
& =\left[\mu_{N_{1}}^{1,-}\left(a_{3} a_{2}\right) \wedge \mu_{N_{1}}^{1,-}\left(a_{2} a_{5}\right)\right] \\
& \vee\left[\mu_{N_{1}}^{1,-}\left(a_{3} a_{4}\right) \wedge \mu_{N_{1}}^{1,-}\left(a_{4} a_{5}\right)\right] \\
& =[0.5 \wedge 0.4] \vee[0.7 \wedge 0.3]=0.4 \text {, } \\
& \mu_{N_{1}}^{3,-}\left(a_{3} a_{4}\right)=\left(\mu^{2,-} n_{N_{1}} \circ \mu_{N_{1}}^{1,-}\right)\left(a_{3} a_{4}\right) \\
& =\left[\mu_{N_{1}}^{2,-}\left(a_{3} a_{2}\right) \wedge \mu_{N_{1}}^{1,-}\left(a_{2} a_{4}\right)\right] \\
& \vee\left[\mu_{N_{1}}^{2,-}\left(a_{3} a_{5}\right) \wedge \mu_{N_{1}}^{1,-}\left(a_{5} a_{4}\right)\right] \\
& =[0.0 \wedge 0.0] \vee[0.4 \wedge 0.3]=0.3 \text {, } \\
& \mu_{N_{1}}^{3,-}\left(a_{3} a_{5}\right)=\left(\mu^{2,-} n_{N_{1}} \circ \mu_{N_{1}}^{1,-}\right)\left(a_{3} a_{5}\right) \\
& =\left[\mu_{N_{1}}^{2,-}\left(a_{3} a_{2}\right) \wedge \mu_{N_{1}}^{1,-}\left(a_{2} a_{5}\right)\right] \\
& \vee\left[\mu_{N_{1}}^{2,-}\left(a_{3} a_{4}\right) \wedge \mu_{N_{1}}^{1,-}\left(a_{4} a_{5}\right)\right] \\
& =[0.0 \wedge 0.4] \vee[0.0 \wedge 0.3]=0.0, \\
& \mu_{N_{1}}^{3,-}\left(a_{4} a_{5}\right)=\left(\mu^{2,-} n_{N_{1}} \circ \mu_{N_{1}}^{1,-}\right)\left(a_{4} a_{5}\right) \\
& =\left[\mu_{N_{1}}^{2,-}\left(a_{4} a_{2}\right) \wedge \mu_{N_{1}}^{1,-}\left(a_{2} a_{5}\right)\right] \\
& \vee\left[\mu_{N_{1}}^{2,-}\left(a_{4} a_{3}\right) \wedge \mu_{N_{1}}^{1,-}\left(a_{3} a_{5}\right)\right] \\
& =[0.5 \wedge 0.4] \vee[0.0 \wedge 0.0]=0.4 \text {, } \\
& \mu_{N_{1}}^{3,-}\left(a_{1} a_{6}\right)=\left(\mu^{2,-} n_{N_{1}} \circ \mu_{N_{1}}^{1,-}\right)\left(a_{1} a_{6}\right)=0 .
\end{aligned}
$$$$
\mu_{N_{1}}^{2,-}\left(a_{4} a_{5}\right)=\left(\mu^{1,-} n_{N_{1}} \circ \mu_{N_{1}}^{1,-}\right)\left(a_{4} a_{5}\right)
$$$$
=\left[\mu_{N_{1}}^{1,-}\left(a_{4} a_{2}\right) \wedge \mu_{N_{1}}^{1,-}\left(a_{2} a_{5}\right)\right]
$$$$
\vee\left[\mu_{N_{1}}^{1,-}\left(a_{4} a_{3}\right) \wedge \mu_{N_{1}}^{1,-}\left(a_{3} a_{5}\right)\right]
$$$$
=[0.0 \wedge 0.4] \vee[0.7 \wedge 0.0]=0.0,
$$$$
\mu_{N_{1}}^{2,-}\left(a_{1} a_{6}\right)=\left(\mu^{1,-} n_{N_{1}} \circ \mu_{N_{1}}^{1,-}\right)\left(a_{1} a_{6}\right)=0,
$$$$
\mu_{N_{1}}^{3,-}\left(a_{2} a_{3}\right)=\left(\mu^{2,-} n_{N_{1}} \circ \mu_{N_{1}}^{1,-}\right)\left(a_{2} a_{3}\right)
$$$$
=\left[\mu_{N_{1}}^{2,-}\left(a_{2} a_{4}\right) \wedge \mu_{N_{1}}^{1,-}\left(a_{4} a_{3}\right)\right]
$$$$
\vee\left[\mu_{N_{1}}^{2,-}\left(a_{2} a_{5}\right) \wedge \mu_{N_{1}}^{1,-}\left(a_{5} a_{3}\right)\right]
$$$$
=[0.5 \wedge 0.7] \vee[0.0 \wedge 0.0]=0.5,
$$$$
\mu_{N_{1}}^{3,-}\left(a_{2} a_{4}\right)=\left(\mu^{2,-} n_{N_{1}} \circ \mu_{N_{1}}^{1,-}\right)\left(a_{2} a_{4}\right)
$$$$
=\left[\mu_{N_{1}}^{2,-}\left(a_{2} a_{3}\right) \wedge \mu_{N_{1}}^{1,-}\left(a_{3} a_{4}\right)\right]
$$$$
\vee\left[\mu_{N_{1}}^{2,-}\left(a_{2} a_{5}\right) \wedge \mu_{N_{1}}^{1,-}\left(a_{5} a_{4}\right)\right]
$$$$
=[0.0 \wedge 0.7] \vee[0.0 \wedge 0.3]=0.0 \text {, }
$$$$
\mu_{N_{1}}^{3,-}\left(a_{2} a_{5}\right)=\left(\mu^{2,-} n_{N_{1}} \circ \mu_{N_{1}}^{1,-}\right)\left(a_{2} a_{5}\right)
$$$$
=\left[\mu_{N_{1}}^{2,-}\left(a_{2} a_{3}\right) \wedge \mu_{N_{1}}^{1,-}\left(a_{3} a_{5}\right)\right]
$$$$
\vee\left[\mu_{N_{1}}^{2,-}\left(a_{2} a_{4}\right) \wedge \mu_{N_{1}}^{1,-}\left(a_{4} a_{5}\right)\right]
$$$$
=[0.0 \wedge 0.0] \vee[0.5 \wedge 0.3]=0.3 \text {, }
$$

Similarly,

$$
\begin{aligned}
& \mu_{N_{1}}^{4,-}\left(a_{2} a_{3}\right)=\left(\mu^{1,-} n_{N_{1}} \circ \mu_{N_{1}}^{1,-}\right)\left(a_{2} a_{3}\right)=0, \\
& \mu_{N_{1}}^{4,-}\left(a_{2} a_{4}\right)=\left(\mu^{1,-} n_{N_{1}} \circ \mu_{N_{1}}^{1,-}\right)\left(a_{2} a_{4}\right)=0.5, \\
& \mu_{N_{1}}^{4,-}\left(a_{2} a_{5}\right)=\left(\mu^{1,-} n_{N_{1}} \circ \mu_{N_{1}}^{1,-}\right)\left(a_{2} a_{5}\right)=0, \\
& \mu_{N_{1}}^{4,-}\left(a_{3} a_{4}\right)=\left(\mu^{1,-} n_{N_{1}} \circ \mu_{N_{1}}^{1,-}\right)\left(a_{3} a_{4}\right)=0, \\
& \mu_{N_{1}}^{4,-}\left(a_{3} a_{5}\right)=\left(\mu^{1,-} n_{N_{1}} \circ \mu_{N_{1}}^{1,-}\right)\left(a_{3} a_{5}\right)=0.4, \\
& \mu_{N_{1}}^{4,-}\left(a_{4} a_{5}\right)=\left(\mu^{1,-} n_{N_{1}} \circ \mu_{N_{1}}^{1,-}\right)\left(a_{4} a_{5}\right)=0, \\
& \mu_{N_{1}}^{4,-}\left(a_{1} a_{6}\right)=\left(\mu^{1,-} n_{N_{1}} \circ \mu_{N_{1}}^{1,-}\right)\left(a_{1} a_{6}\right)=0 .
\end{aligned}
$$

This implies that

$$
\begin{aligned}
& \mu_{N_{1}}^{\infty,-}\left(a_{2} a_{3}\right)=\vee\{0.5,0.0,0.5,0.0\}=0.5, \\
& \mu_{N_{1}}^{\infty,-}\left(a_{2} a_{4}\right)=\vee\{0.0,0.5,0.0,0.5\}=0.5, \\
& \mu_{N_{1}}^{\infty,-}\left(a_{2} a_{5}\right)=\vee\{0.4,0.0,0.3,0.0\}=0.4, \\
& \mu_{N_{1}}^{\infty,-}\left(a_{3} a_{4}\right)=\vee\{0.7,0.0,0.3,0.0\}=0.7, \\
& \mu_{N_{1}}^{\infty,-}\left(a_{3} a_{5}\right)=\vee\{0.0,0.4,0.0,0.4\}=0.4, \\
& \mu_{N_{1}}^{\infty,-}\left(a_{4} a_{5}\right)=\vee\{0.3,0.0,0.4,0.0\}=0.4, \\
& \mu_{N_{1}}^{\infty,-}\left(a_{1} a_{6}\right)=\vee\{0.3,0.0,0.0,0.0\}=0.2 .
\end{aligned}
$$

For all the remaining pairs of vertices, $N_{1}$-loss and $N_{1}$-gain of connectedness are zero. 
Definition 29. A BFGS $\check{G}_{b}=\left(M, N_{1}, N_{2}, \ldots, N_{n}\right)$ of a graph structure $G^{*}=\left(U, E_{1}, E_{2}, \ldots, E_{n}\right)$ is an $N_{i}$-cycle if $\left(\operatorname{supp}(M), \operatorname{supp}\left(N_{1}\right), \operatorname{supp}\left(N_{2}\right), \ldots, \operatorname{supp}\left(N_{n}\right)\right)$ is an $E_{i}-$ cycle.

Definition 30. A BFGS $\check{G}_{b}=\left(M, N_{1}, N_{2}, \ldots, N_{n}\right)$ of a graph structure $G^{*}=\left(U, E_{1}, E_{2}, \ldots, E_{n}\right)$ is a bipolar fuzzy $N_{i}$-cycle for some $i$ if

(i) $\check{G}_{b}$ is an $N_{i}$-cycle;

(ii) there is no unique $N_{i}$-edge $u v$ in $\check{G}_{b}$ such that $\mu_{N_{i}}^{P}(u v)=\min \left\{\mu_{N_{i}}^{P}(x y): x y \in E_{i}=\operatorname{supp}\left(N_{i}\right)\right\}$ or $\mu_{N_{i}}^{N}(u v)=\max \left\{\mu_{N_{i}}^{N}(x y): x y \in E_{i}=\operatorname{supp}\left(N_{i}\right)\right\}$.

Example 31. Consider BFGS $\check{G}_{b}=\left(M, N_{1}, N_{2}\right)$ as shown in Figure 3. Then $\check{G}_{b}$ is an $N_{1}$-cycle as well as bipolar fuzzy $N_{1}$-cycle, since $\left(\operatorname{supp}(M), \operatorname{supp}\left(N_{1}\right), \operatorname{supp}\left(N_{2}\right)\right)$ is an $E_{1}$-cycle and there are two $N_{1}$-edges with minimum positive degree and more than one $N_{1}$-edge with maximum negative degree of all $N_{1}$-edges.

Definition 32. Let $\check{G}_{b}=\left(M, N_{1}, N_{2}, \ldots, N_{n}\right)$ be a BFGS of a graph structure $G^{*}=\left(U, E_{1}, E_{2}, \ldots, E_{n}\right)$ and $x$ a vertex of $\check{G}_{b}$. Let $\left(M^{\prime}, N_{1}^{\prime}, N_{2}^{\prime}, \ldots, N_{n}^{\prime}\right)$ be a bipolar fuzy subgraph structure of $\check{G}_{b}$ induced by $U \backslash\{x\}$ such that

$$
\begin{aligned}
& \mu_{M^{\prime}}^{P}(x)=0=\mu_{M^{\prime}}^{N}(x), \\
& \mu_{N_{i}^{\prime}}^{P}(x v)=0=\mu_{N_{i}^{\prime}}^{N}(x v) \\
& \quad \forall \text { edges } x v \in \check{G}_{b}, \\
& \mu_{M^{\prime}}^{P}(v)=\mu_{M}^{P}(v), \\
& \mu_{M^{\prime}}^{N}(v)=\mu_{M}^{N}(v),
\end{aligned}
$$

$\forall v \neq x$,

$$
\begin{aligned}
& \mu_{N_{i}^{\prime}}^{P}(u v)=\mu_{N_{i}}^{P}(u v), \\
& \mu_{N_{i}^{\prime}}^{N}(u v)=\mu_{N_{i}}^{N}(u v)
\end{aligned}
$$

$\forall i$, such that $u \neq x, v \neq x$.

Then $x$ is a bipolar fuzzy $N_{i}$-cut vertex for some $i$, if

$$
\begin{aligned}
& \mu_{N_{i}}^{\infty,+}(u v)>\mu_{N_{i}^{\prime}}^{\infty,+}(u v), \\
& \mu_{N_{i}}^{\infty,-}(u v)>\mu_{N_{i}^{\prime}}^{\infty,-}(u v)
\end{aligned}
$$

for some $u, v \in U \backslash\{x\}$.

And, $x$ is an $N_{i}-P$ bipolar fuzzy cut vertex if only the first condition holds and a $N_{i}-N$ bipolar fuzzy cut vertex if only the second condition holds.

Example 33. Consider BFSG $\check{G}_{b}=\left(M, N_{1}, N_{2}\right)$ as considered in Example 28 and shown in Figure 5; after deleting vertex $a_{2}$, the resulting bipolar fuzzy subgraph structure will be as shown in Figure 6.

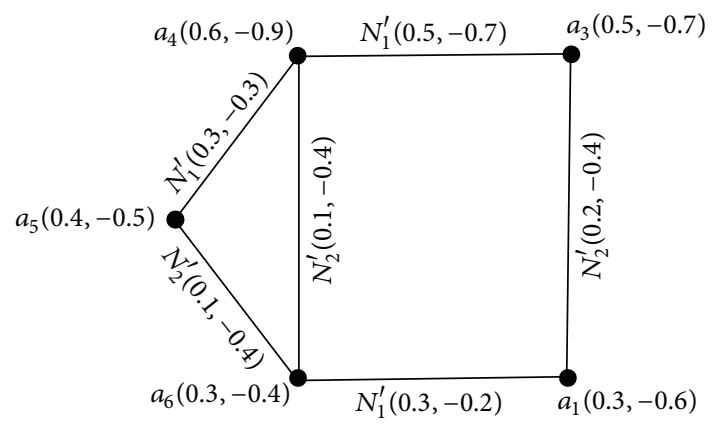

Figure 6: Bipolar fuzzy subgraph structure $\left(M \backslash\left\{a_{2}\right\}, N_{1}^{\prime}, N_{2}^{\prime}\right)$.

Then $a_{2}$ is a bipolar fuzzy $N_{1}-N$ cut vertex since

$$
\begin{aligned}
& \mu_{N_{1}}^{\infty,-}\left(a_{3} a_{4}\right)=0.7=\mu_{N_{1}^{\prime}}^{\infty,-}\left(a_{3} a_{4}\right), \\
& \mu_{N_{1}}^{\infty,-}\left(a_{3} a_{5}\right)=0.4>0.3=\mu_{N_{1}}^{\infty,-}\left(a_{3} a_{5}\right), \\
& \mu_{N_{1}}^{\infty,-}\left(a_{4} a_{5}\right)=0.4>0.3=\mu_{N_{1}^{\prime}}^{\infty,-}\left(a_{4} a_{5}\right), \\
& \mu_{N_{1}}^{\infty,-}\left(a_{1} a_{6}\right)=0.2=\mu_{N_{1}}^{\infty,-}\left(a_{1} a_{6}\right) .
\end{aligned}
$$

Definition 34. Let $\check{G}_{b}=\left(M, N_{1}, N_{2}, \ldots, N_{n}\right)$ be a BFGS of a graph structure $G^{*}=\left(U, E_{1}, E_{2}, \ldots, E_{n}\right)$ and let $x y$ be an $N_{i}$-edge. Let $\left(M, N_{1}^{\prime}, N_{2}^{\prime}, \ldots, N_{n}^{\prime}\right)$ be a bipolar fuzzy spanning subgraph structure of $\check{G}_{b}$, obtained by taking

$$
\begin{aligned}
& \mu_{N_{i}^{\prime}}^{P}(x y)=0=\mu_{N_{i}^{\prime}}^{N}(x y), \\
& \mu_{N_{i}^{\prime}}^{P}(u v)=\mu_{N_{i}}^{P}(u v), \\
& \mu_{N_{i}^{\prime}}^{N}(u v)=\mu_{N_{i}}^{N}(u v)
\end{aligned}
$$

$\forall$ edges $u v \neq x y$.

Then $x y$ is a bipolar fuzzy $N_{i}$-bridge if

$$
\begin{aligned}
& \mu_{N_{i}}^{\infty,+}(u v)>\mu_{N_{i}^{\prime}}^{\infty,+}(u v), \\
& \mu_{N_{i}}^{\infty,-}(u v)>\mu_{N_{i}^{\prime}}^{\infty,-}(u v)
\end{aligned}
$$

for some $u, v \in U$.

Edge $x y$ is an $N_{i}-P$ bipolar fuzzy bridge if only the first condition holds and an $N_{i}-N$ bipolar fuzzy bridge if only the second condition holds.

Example 35. Consider the BFGS $\check{G}_{b}=\left(M, N_{1}, N_{2}\right)$ as shown in Figure 6 and let $\check{G}_{b}^{\prime}=\left(M, N_{1}^{\prime}, N_{2}^{\prime}\right)$ be bipolar fuzzy spanning subgraph structure of $\check{G}_{b}$ obtained by deleting $N_{1}$ edge $\left(a_{2} a_{5}\right)$. Then $a_{2} a_{5}$ is a bipolar fuzzy $N_{1}$-bridge, since $\mu_{N_{1}}^{\infty,+}\left(a_{2} a_{5}\right)=0.4>0.3=\mu_{N_{1}^{\prime}}^{\infty,+}\left(a_{2} a_{5}\right)$ and $\mu_{N_{1}}^{\infty,-}\left(a_{2} a_{5}\right)=0.4>$ $0.3=\mu_{N_{1}^{\prime}}^{\infty,-}\left(a_{2} a_{5}\right)$, and also abipolar fuzzy $N_{1}-N$ bridge, since $\mu_{N_{1}}^{\infty,-}\left(a_{3} a_{5}\right)=0.4>0.3=\mu_{N_{1}^{\prime}}^{\infty,-}\left(a_{3} a_{5}\right)$ and $\mu_{N_{1}}^{\infty,-}\left(a_{4} a_{5}\right)=0.4>$ $0.3=\mu_{N_{1}^{\prime}}^{\infty,-}\left(a_{4} a_{5}\right)$. 


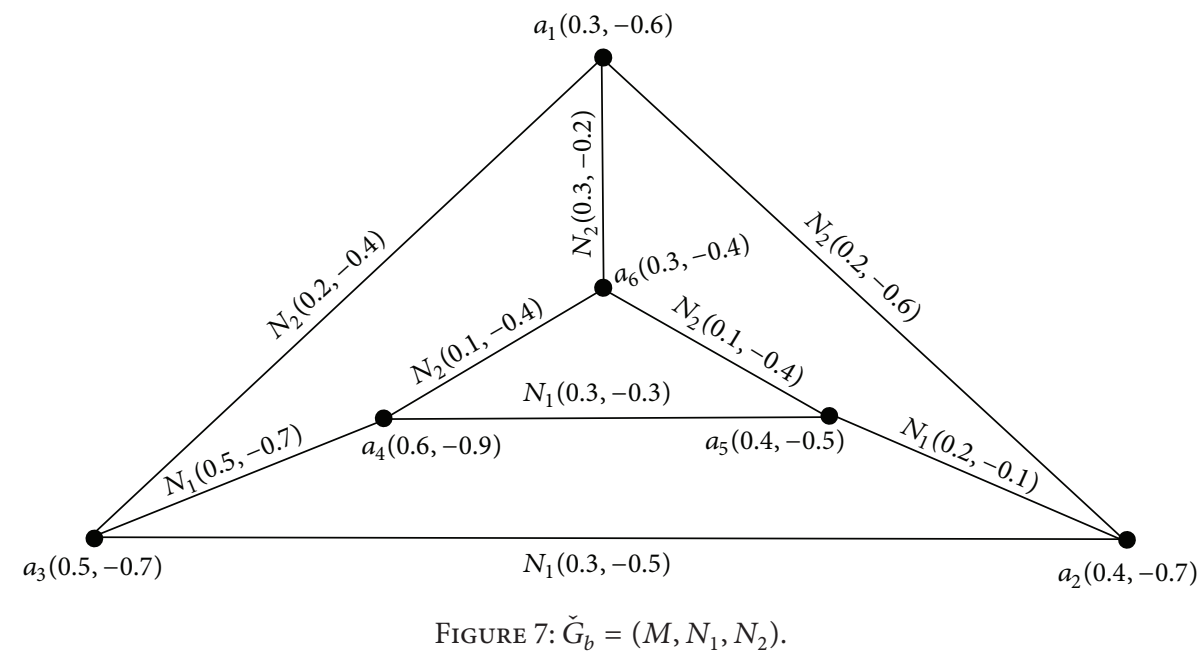

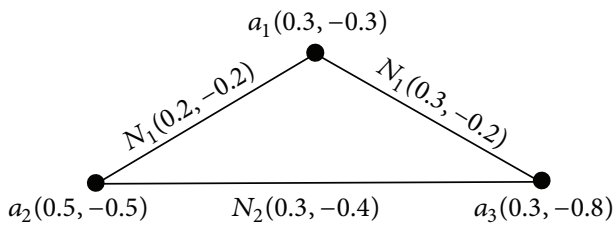

(BFGS $\left.\check{G}_{b 1}\right)$

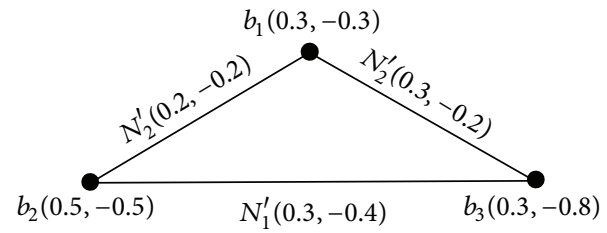

(BFGS $\left.\check{G}_{b 2}\right)$

FIGURE 8: Isomorphic bipolar fuzzy graph structures.

Definition 36. A BFGS $\check{G}_{b}=\left(M, N_{1}, N_{2}, \ldots, N_{n}\right)$ of a graph structure $G^{*}=\left(U, E_{1}, E_{2}, \ldots, E_{n}\right)$ is an $N_{i}$-tree if $\left(\operatorname{supp}(A), \operatorname{supp}\left(N_{1}\right), \operatorname{supp}\left(N_{2}\right), \ldots, \operatorname{supp}\left(N_{n}\right)\right)$ is an $E_{i}$-tree. In other words, $\check{G}_{b}$ is an $N_{i}$-tree if a subgraph of $\check{G}_{b}$, induced by $\operatorname{supp}\left(N_{i}\right)$, forms a tree.

Definition 37. A BFGS $\check{G}_{b}=\left(M, N_{1}, N_{2}, \ldots, N_{n}\right)$ of a graph structure $G^{*}=\left(U, E_{1}, E_{2}, \ldots, E_{n}\right)$ is a bipolar fuzzy $N_{i}$-tree if $\check{G}_{b}$ has a bipolar fuzzy spanning subgraph structure $\check{H}_{b}=$ $\left(A, C_{1}, C_{2}, \ldots, C_{n}\right)$ such that $\check{H}_{b}$ is a $C_{i}$-tree and $\mu_{N_{i}}^{P}(x y)<$ $\mu_{C_{i}}^{\infty,+}(x y)$ and $\left|\mu_{N_{i}}^{N}(x y)\right|<\mu_{C_{i}}^{\infty,-}(x y) \forall N_{i}$-edges not in $\check{H}_{b}$.

In more concerned view, $\check{G}_{b}$ is a bipolar fuzzy $N_{i}-P$ tree if only the first condition holds and a bipolar fuzzy $N_{i}-N$ tree if only the second condition holds.

Example 38. Consider BFGS $\check{G}_{b}=\left(M, N_{1}, N_{2}\right)$ as shown in Figure 7, which is an $N_{2}$-tree. It is not an $N_{1}$-tree but a bipolar fuzzy $N_{1}$-tree since it has a bipolar fuzzy spanning subgraph structure $\left(M, N_{1}^{\prime}, N_{2}^{\prime}\right)$ as an $N_{1}$-tree, which is obtained by deleting $N_{1}$-edge $a_{2} a_{5}$ from $\check{G}_{b}$ and

$$
\begin{gathered}
\mu_{N_{1}}^{P}\left(a_{2} a_{5}\right)=0.2<0.3=\mu_{N_{1}^{\prime}}^{\infty,+}\left(a_{2} a_{5}\right), \\
\left|\mu_{N_{1}}^{N}\left(a_{2} a_{5}\right)\right|=0.1<0.3=\mu_{N_{1}^{\prime}}^{\infty,-}\left(a_{2} a_{5}\right) .
\end{gathered}
$$

Definition 39. A BFGS $\check{G}_{s 1}=\left(M_{1}, N_{11}, N_{12}, \ldots, N_{1 n}\right)$ of graph structure $G_{1}^{*}=\left(U_{1}, E_{11}, E_{12}, \ldots, E_{1 n}\right)$ is isomorphic to a BFGS $\check{G}_{s 2}=\left(M_{2}, N_{21}, N_{22}, \ldots, N_{2 n}\right)$ of $G_{2}^{*}=$
$\left(U_{2}, E_{21}, E_{22}, \ldots, E_{2 n}\right)$ if there exists a bijection $f: U_{1} \rightarrow U_{2}$ and a permutation $\phi$ on the set $\{1,2, \ldots, n\}$ such that

$$
\begin{aligned}
& \mu_{M_{1}}^{P}\left(u_{1}\right)=\mu_{M_{2}}^{P}\left(f\left(u_{1}\right)\right) \\
& \mu_{M_{1}}^{N}\left(u_{1}\right)=\mu_{M_{2}}^{N}\left(f\left(u_{1}\right)\right)
\end{aligned}
$$

$\forall u_{1} \in U_{1}$

and for $\phi(i)=j$

$$
\begin{aligned}
& \mu_{N_{1 i}}^{P}\left(u_{1} u_{2}\right)=\mu_{N_{2 j}}^{P}\left(f\left(u_{1}\right) f\left(u_{2}\right)\right), \\
& \mu_{N_{1 i}}^{N}\left(u_{1} u_{2}\right)=\mu_{N_{2 j}}^{N}\left(f\left(u_{1}\right) f\left(u_{2}\right)\right) \\
& \forall u_{1} u_{2} \in E_{1 i}, i=1,2, \ldots, n .
\end{aligned}
$$

Example 40. Let $\check{G}_{b 1}=\left(M, N_{1}, N_{2}\right)$ and $\check{G}_{b 2}=\left(M^{\prime}, N_{1}^{\prime}, N_{2}^{\prime}\right)$ be two BFGSs of graph structures $G_{1}^{*}=\left(U, E_{1}, E_{2}\right)$ and $G_{2}^{*}=$ $\left(U^{\prime}, E_{1}^{\prime}, E_{2}^{\prime}\right)$, respectively, as shown in Figure 8.

Here $\check{G}_{b 1}$ is isomorphic (not identical) to $\check{G}_{b 2}$ under the mapping $f: U \rightarrow U^{\prime}$, defined by $f\left(a_{1}\right)=b_{1}, f\left(a_{2}\right)=b_{2}$, and $f\left(a_{3}\right)=b_{3}$, and a permutation $\phi$ given by $\phi(1)=2, \phi(2)=1$, such that

$$
\begin{aligned}
& \mu_{M}^{P}\left(a_{i}\right)=\mu_{M^{\prime}}^{P}\left(f\left(a_{i}\right)\right), \\
& \mu_{M}^{N}\left(a_{i}\right)=\mu_{M^{\prime}}^{N}\left(f\left(a_{i}\right)\right)
\end{aligned}
$$

$\forall a_{i} \in U$, 


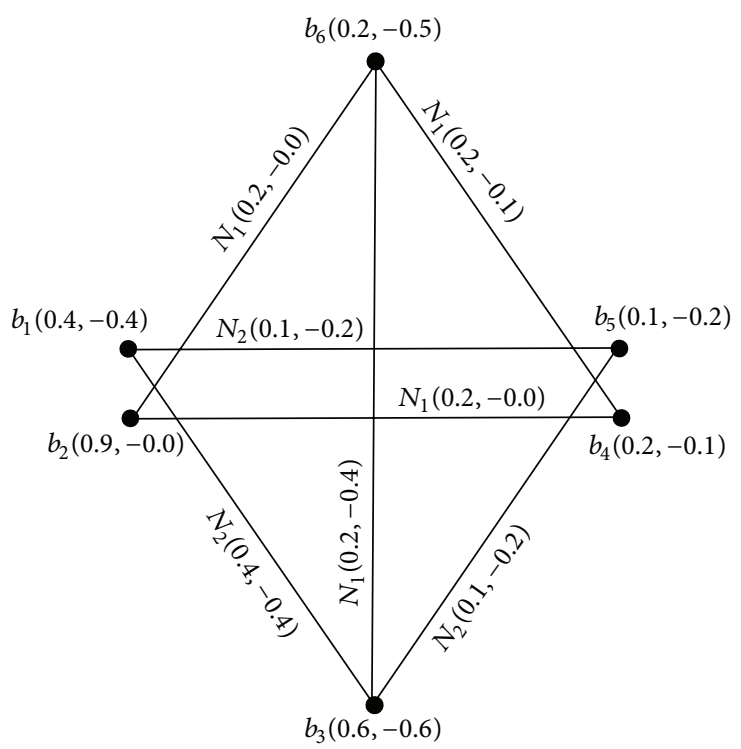

$\left(\check{G}_{b 1}\right)$

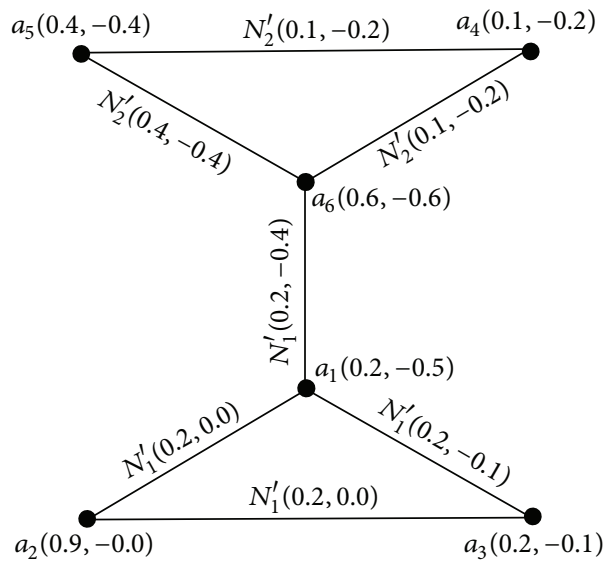

$\left(\check{G}_{b 2}\right)$

FIGURE 9: Identical bipolar fuzzy graph structures.

$$
\begin{aligned}
& \mu_{N_{k}}^{P}\left(a_{i} a_{j}\right)=\mu_{N_{\phi(k)}}^{P}\left(f\left(a_{i}\right) f\left(a_{j}\right)\right), \\
& \mu_{N_{k}}^{N}\left(a_{i} a_{j}\right)=\mu_{N_{\phi(k)}}^{N}\left(f\left(a_{i}\right) f\left(a_{j}\right)\right)
\end{aligned}
$$$$
\forall a_{i} a_{j} \in E_{k}, \quad k=1,2 .
$$

Definition 41. A BFGS $\check{G}_{s 1}=\left(M_{1}, N_{11}, N_{12}, \ldots, N_{1 n}\right)$ of GS $G_{1}^{*}=\left(U, E_{11}, E_{12}, \ldots, E_{1 n}\right)$ is identical to a BFGS $\check{G}_{s 2}=$ $\left(M_{2}, N_{21}, N_{22}, \ldots, N_{2 n}\right)$ of GS $G_{2}^{*}=\left(U, E_{21}, E_{22}, \ldots, E_{2 n}\right)$ if there exist a bijection $f: U \rightarrow U$, such that

$$
\begin{aligned}
& \mu_{M_{1}}^{P}(u)=\mu_{M_{2}}^{P}(f(u)), \\
& \mu_{M_{1}}^{N}(u)=\mu_{M_{2}}^{N}(f(u))
\end{aligned}
$$

$\forall u \in U$,

$$
\begin{aligned}
& \mu_{N_{1 i}}^{P}\left(u_{1} u_{2}\right)=\mu_{N_{2 i}}^{P}\left(f\left(u_{1}\right) f\left(u_{2}\right)\right), \\
& \mu_{N_{1 i}}^{N}\left(u_{1} u_{2}\right)=\mu_{N_{2 i}}^{N}\left(f\left(u_{1}\right) f\left(u_{2}\right)\right)
\end{aligned}
$$

$$
\forall u_{1} u_{2} \in E_{1 i}, \quad i=1,2, \ldots, n .
$$

Example 42. Let $\check{G}_{b 1}=\left(M, N_{1}, N_{2}\right)$ and $\check{G}_{b 2}=\left(M^{\prime}, N_{1}^{\prime}, N_{2}^{\prime}\right)$ be two BFGSs of graph structures $G_{1}^{*}=\left(U, E_{1}, E_{2}\right)$ and $G_{2}^{*}=$ $\left(U^{\prime}, E_{1}^{\prime}, E_{2}^{\prime}\right)$, respectively, as shown in Figure 9.

Here $\check{G}_{b 1}$ is identical with $\check{G}_{b 2}$ under the mapping $f$ : $U \rightarrow U^{\prime}$, defined by $f\left(a_{1}\right)=b_{6}, f\left(a_{2}\right)=b_{2}, f\left(a_{3}\right)=$ $b_{4}, f\left(a_{4}\right)=b_{5}, f\left(a_{5}\right)=b_{1}$, and $f\left(a_{6}\right)=b_{3}$, such that

$$
\begin{aligned}
& \mu_{M}^{P}\left(a_{i}\right)=\mu_{M^{\prime}}^{P}\left(f\left(a_{i}\right)\right), \\
& \mu_{M}^{N}\left(a_{i}\right)=\mu_{M^{\prime}}^{N}\left(f\left(a_{i}\right)\right)
\end{aligned}
$$

$\forall a_{i} \in U$

$$
\begin{aligned}
& \mu_{N_{k}}^{P}\left(a_{i} a_{j}\right)=\mu_{N_{k}^{\prime}}^{P}\left(f\left(a_{i}\right) f\left(a_{j}\right)\right), \\
& \mu_{N_{k}}^{N}\left(a_{i} a_{j}\right)=\mu_{N_{k}^{\prime}}^{N}\left(f\left(a_{i}\right) f\left(a_{j}\right)\right)
\end{aligned}
$$$$
\forall a_{i} a_{j} \in E_{k}, k=1,2 .
$$

Definition 43. Let $\check{G}_{b}=\left(M, N_{1}, N_{2}, \ldots, N_{n}\right)$ be a BFGS of a GS $G^{*}=\left(U, E_{1}, E_{2}, \ldots, E_{n}\right)$. Let $\phi$ be any permutation on the set $\left\{E_{1}, E_{2}, \ldots, E_{n}\right\}$ and the corresponding permutation on $\left\{N_{1}, N_{2}, \ldots, N_{n}\right\}$; that is, $\phi\left(N_{i}\right)=N_{j}$ if and only if $\phi\left(E_{i}\right)=$ $E_{j} \forall i$.

If $x y \in N_{r}$ for some $r$ and

$$
\begin{aligned}
& \mu_{N_{i}^{\phi}}^{P}(x y)=\mu_{M}^{P}(x) \wedge \mu_{M}^{P}(y)-\bigvee_{j \neq i} \mu_{\phi N_{j}}^{P}(x y), \\
& \mu_{N_{i}^{\phi}}^{N}(x y)=\mu_{M}^{N}(x) \vee \mu_{M}^{N}(y)-\bigwedge_{j \neq i} \mu_{\phi N_{j}}^{N}(x y), \\
& \quad i=1,2, \ldots, n,
\end{aligned}
$$

then $x y \in B_{m}^{\phi}$, while $m$ is chosen such that $\mu_{N_{m}^{\phi}}^{P}(x y) \geq$ $\mu_{N_{i}^{\phi}}^{P}(x y)$ and $\mu_{N_{m}^{\phi}}^{N}(x y) \leq \mu_{N_{i}^{\phi}}^{N}(x y) \forall i$.

And BFGS $\left(M, N_{1}^{\phi}, N_{2}^{\phi}, \ldots, N_{n}^{\phi}\right)$, denoted by $\check{G}_{b}^{\phi c}$, is called the $\phi$-complement of BFGS $\check{G}_{b}$.

Example 44. Let $M=\left\{\left(a_{1}, 0.3,-0.7\right),\left(a_{2}, 0.5,-0.4\right)\right.$, $\left.\left(a_{3}, 0.7,-0.3\right)\right\}, N_{1}=\left\{\left(a_{1} a_{3}, 0.3,-0.3\right),\left(a_{2} a_{3}, 0.5,-0.3\right)\right\}$, and $N_{2}=\left\{\left(a_{1} a_{2}, 0.3,-0.4\right)\right\}$ be bipolar fuzzy subsets of $U, E_{1}$, and $E_{2}$, respectively, so that $\check{G}_{b}=\left(M, N_{1}, N_{2}\right)$ is a BFGS of graph structure $G^{*}=\left(U, E_{1}, E_{2}\right)$. Let $\phi$ be a permutation on the set $\left\{N_{1}, N_{2}\right\}$ such that $\phi\left(N_{1}\right)=N_{2}$ and $\phi\left(N_{2}\right)=N_{1}$. 
Now for $a_{2} a_{3} \in N_{1}$,

$$
\begin{aligned}
\mu_{N_{1}^{\phi}}^{P}\left(a_{2} a_{3}\right) & =\mu_{M}^{P}\left(a_{2}\right) \wedge \mu_{M}^{P}\left(a_{3}\right)-\bigvee_{j \neq 1}\left[\mu_{\phi N_{j}}^{P}\left(a_{2} a_{3}\right)\right] \\
& =0.5 \wedge 0.7-\left[\mu_{\phi N_{2}}^{P}\left(a_{2} a_{3}\right)\right] \\
& =0.5-\mu_{N_{1}}^{P}\left(a_{2} a_{3}\right)=0.5-0.5=0, \\
\mu_{N_{1}^{\phi}}^{N}\left(a_{2} a_{3}\right) & =\mu_{M}^{N}\left(a_{2}\right) \vee \mu_{M}^{N}\left(a_{3}\right)-\bigwedge_{j \neq 1}\left[\mu_{\phi N_{j}}^{N}\left(a_{2} a_{3}\right)\right] \\
& =-0.4 \vee-0.3-\left[\mu_{\phi N_{2}}^{N}\left(a_{2} a_{3}\right)\right] \\
& =-0.3-\mu_{N_{1}}^{N}\left(a_{2} a_{3}\right)=-0.3+0.3=0, \\
\mu_{N_{2}^{\phi}}^{P}\left(a_{2} a_{3}\right) & =\mu_{M}^{P}\left(a_{2}\right) \wedge \mu_{M}^{P}\left(a_{3}\right)-\bigvee_{j \neq 2}\left[\mu_{\phi N_{j}}^{P}\left(a_{2} a_{3}\right)\right] \\
& =0.5 \wedge 0.7-\left[\mu_{\phi N_{1}}^{P}\left(a_{2} a_{3}\right)\right] \\
& =0.5-\mu_{N_{2}}^{P}\left(a_{2} a_{3}\right)=0.5-0=0.5, \\
& =-0.3 \vee-0.3-\left[\mu_{N_{2}}^{N}\left(a_{2} a_{3}\right)=-0.3-0=-0.3 .\right. \\
\mu_{N_{2}^{\phi}}^{N}\left(a_{2} a_{3}\right) & =\mu_{M}^{N}\left(a_{2}\right) \vee \mu_{M}^{N}\left(a_{3}\right)-\bigwedge_{j \neq 2}\left[\mu_{\phi N_{j}}^{N}\left(a_{2} a_{3}\right)\right] \\
& \\
& =0.4
\end{aligned}
$$

Clearly, $\mu_{\phi N_{2}}^{P}\left(a_{2} a_{3}\right)=0.5>0=\mu_{\phi N_{1}}^{P}\left(a_{2} a_{3}\right)$ and $\mu_{\phi N_{2}}^{N}\left(a_{2} a_{3}\right)=$ $-0.3<0=\mu_{\phi N_{1}}^{N}\left(a_{2} a_{3}\right)$. So $a_{2} a_{3} \in N_{2}^{\phi}$.

Similarly for $a_{1} a_{3} \in N_{1}, \mu_{N_{1}^{\phi}}^{P}\left(a_{1} a_{3}\right)=0, \mu_{N_{1}^{\phi}}^{N}\left(a_{1} a_{3}\right)=$ $0, \mu_{N_{2}^{\phi}}^{P}\left(a_{1} a_{3}\right)=0.3$, and $\mu_{N_{2}^{\phi}}^{N}\left(a_{1} a_{3}\right)=-0.3$.

$\Rightarrow \mu_{N_{2}^{\phi}}^{P}\left(a_{1} a_{3}\right)=0.3>0=\mu_{N_{1}^{\phi}}^{P}\left(a_{1} a_{3}\right)$ and $\mu_{N_{2}^{\phi}}^{N}\left(a_{1} a_{3}\right)=$ $-0.3<0=\mu_{N_{1}^{\phi}}^{N}\left(a_{1} a_{3}\right)$. So $a_{1} a_{3} \in N_{2}^{\phi}$.

And for $a_{1} a_{2} \in N_{2}, \mu_{N_{1}^{\phi}}^{P}\left(a_{1} a_{2}\right)=0.3, \mu_{N_{1}^{\phi}}^{N}\left(a_{1} a_{2}\right)=-0.4$, $\mu_{N_{2}^{\phi}}^{P}\left(a_{1} a_{2}\right)=0$, and $\mu_{N_{2}^{\phi}}^{N}\left(a_{1} a_{2}\right)=0$.

$\Rightarrow \mu_{N_{1}^{\phi}}^{P}\left(a_{1} a_{2}\right)=0.3>0=\mu_{N_{2}^{\phi}}^{P}\left(a_{1} a_{2}\right)$ and $\mu_{N_{1}^{\phi}}^{N}\left(a_{1} a_{2}\right)=$ $-0.4<0=\mu_{N_{2}^{\phi}}^{N}\left(a_{1} a_{2}\right)$. So $a_{1} a_{2} \in N_{1}^{\phi}$.

This implies that

$$
\begin{aligned}
& N_{1}^{\phi}=\left\{\left(a_{1} a_{2}, 0.3,-0.4\right)\right\}, \\
& N_{2}^{\phi}=\left\{\left(a_{2} a_{3}, 0.5,-0.3\right),\left(a_{1} a_{3}, 0.3,-0.3\right)\right\}
\end{aligned}
$$

and $\check{G}_{b}^{\phi c}=\left(M, N_{1}^{\phi}, N_{2}^{\phi}\right)$ is the $\phi$-complement of $\check{G}_{b}$.

Theorem 45. A $\phi$-complement of a bipolar fuzzy graph structure is always a strong BFGS. Moreover, if $\phi(i)=r$ for $r, i \in\{1,2, \ldots, n\}$, then all $N_{r}$-edges in BFGS $\check{G}_{b}=$ $\left(M, N_{1}, N_{2}, \ldots, N_{n}\right)$ become $B_{i}^{\phi}$-edges in $\check{G}_{b}^{\phi c}=\left(A, B_{1}^{\phi}, B_{2}^{\phi}\right.$, $\left.\ldots, B_{n}^{\phi}\right)$.
Proof. From the definition of $\phi$-complement $\check{G}_{b}^{\phi c}$,

$$
\begin{aligned}
& \mu_{N_{i}^{\phi}}^{P}(x y)=\mu_{M}^{P}(x) \wedge \mu_{M}^{P}(y)-\bigvee_{j \neq i} \mu_{\phi N_{j}}^{P}(x y), \\
& \mu_{N_{i}^{\phi}}^{N}(x y)=\mu_{M}^{N}(x) \vee \mu_{M}^{N}(y)-\bigwedge_{j \neq i} \mu_{\phi N_{j}}^{N}(x y),
\end{aligned}
$$

for $i=1,2, \ldots, n$.

Let us consider expression (37) first.

Since $\mu_{M}^{N}(x) \vee \mu_{M}^{N}(y) \leq 0$ and $\bigwedge_{j \neq i} \mu_{\phi N_{j}}^{N}(x y) \leq 0$, we can write

$$
\mu_{N_{i}^{\phi}}^{N}(x y)=-\left|\mu_{M}^{N}(x) \vee \mu_{M}^{N}(y)\right|+\left|\bigwedge_{j \neq i} \mu_{\phi N_{j}}^{N}(x y)\right| .
$$

Also from the definition of a BFGS $\mu_{N_{j}}^{N}(x y) \geq \mu_{M}^{N}(x) \vee$ $\mu_{M}^{N}(y) \forall N_{j}$

$$
\begin{gathered}
\Longrightarrow \bigwedge_{j \neq i} \mu_{\phi N_{j}}^{N}(x y) \geq \mu_{M}^{N}(x) \vee \mu_{M}^{N}(y) \Longrightarrow \\
\left|\bigwedge_{j \neq i} \mu_{\phi N_{j}}^{N}(x y)\right| \leq\left|\mu_{M}^{N}(x) \vee \mu_{M}^{N}(y)\right| \Longrightarrow \\
-\left|\mu_{M}^{N}(x) \vee \mu_{M}^{N}(y)\right|+\left|\bigwedge_{j \neq i} \mu_{\phi N_{j}}^{N}(x y)\right| \leq 0 .
\end{gathered}
$$

Therefore, $\mu_{N_{i}^{\phi}}^{N}(x y) \leq 0 \forall i$.

Now a requirement is minimum value of $\mu_{N_{i}^{\phi}}^{N}(x y)$. Since $\mu_{N_{i}^{\phi}}^{N}(x y) \leq 0$, that is why it is minimum when its positive part $\left|\bigwedge_{j \neq i} \mu_{\phi N_{j}}^{N}(x y)\right|$ is zero. And $\left|\bigwedge_{j \neq i} \mu_{\phi N_{j}}^{N}(x y)\right|=0$ when $\phi N_{i}=$ $N_{r}$ and $x y$ is an $N_{r}$-edge. So

$$
\mu_{N_{i}^{\phi}}^{N}(x y)=\mu_{M}^{N}(x) \vee \mu_{M}^{N}(y),
$$

$$
\text { for } x y \in N_{r}, \phi N_{i}=N_{r} \text {. }
$$

Similarly for expression (38), a requirement is maximum value of $\mu_{N_{i}^{\phi}}^{P}(x y)$. Since $\mu_{M}^{P}(x) \wedge \mu_{M}^{P}(y) \geq 0, \bigvee_{j \neq i} \mu_{\phi N_{j}}^{P}(x y) \geq 0$ and $\mu_{N_{j}}^{P}(x y) \leq \mu_{M}^{P}(x) \wedge \mu_{M}^{P}(y) \forall N_{j}$

$$
\Longrightarrow \bigvee_{j \neq i} \mu_{\phi N_{j}}^{P}(x y) \leq \mu_{M}^{P}(x) \wedge \mu_{M}^{P}(y) \Longrightarrow
$$

$$
\mu_{M}^{P}(x) \wedge \mu_{M}^{P}(y)-\bigvee_{j \neq i} \mu_{\phi N_{j}}^{P}(x y) \geq 0 .
$$

Therefore, $\mu_{N_{i}^{\phi}}^{P}(x y) \geq 0 \forall i$.

Now $\mu_{N_{i}^{\phi}}^{P}(x y)$ will be maximum when its negative part $\left[-\bigvee_{j \neq i} \mu_{\phi N_{j}}^{P}(x y)\right]$ becomes zero. Clearly, $\left[-\bigvee_{j \neq i} \mu_{\phi N_{j}}^{P}(x y)\right]=0$ when $\phi N_{i}=N_{r}$ and $x y$ is an $N_{r}$-edge. So

$$
\mu_{N_{i}^{\phi}}^{P}(x y)=\mu_{M}^{P}(x) \wedge \mu_{M}^{P}(y) \text {, }
$$

for $x y \in N_{r}, \phi N_{i}=N_{r}$.

From (41) and (43), the conclusion is obvious. 


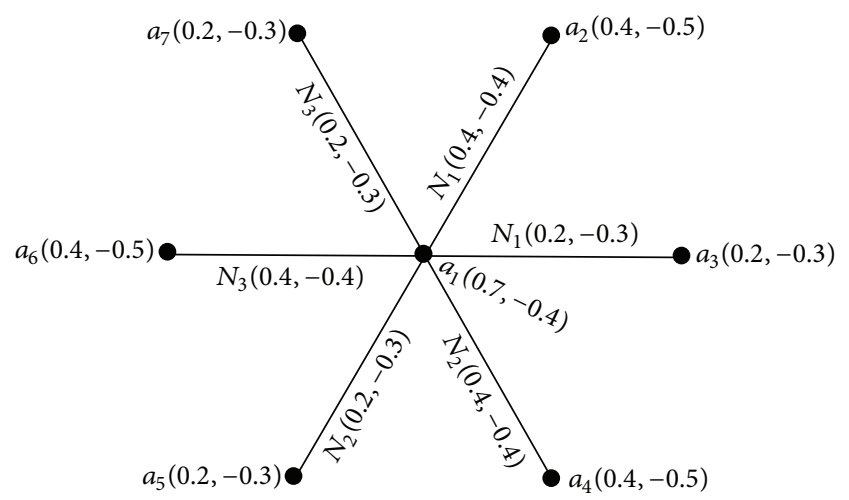

FIGURE 10: Totally strong self-complement BFGS.

Definition 46. Let $\check{G}_{b}=\left(M, N_{1}, N_{2}, \ldots, N_{n}\right)$ be a BFGS and let $\phi$ be any permutation on the set $\{1,2, \ldots, n\}$. Then

(i) $\check{G}_{b}$ is self-complement if it is isomorphic to $\check{G}_{b}^{\phi c}$, the $\phi$ complement of $\check{G}_{b}$;

(ii) $\check{G}_{b}$ is strong self-complement if it is identical to $\check{G}_{b}^{\phi c}$.

Definition 47. Let $\check{G}_{b}=\left(M, N_{1}, N_{2}, \ldots, N_{n}\right)$ be a BFGS. Then

(i) $\check{G}_{b}$ is totally self-complement if it is isomorphic to $\check{G}_{b}^{\phi c}$, the $\phi$-complement of $\check{G}_{b}$, for all permutations $\phi$ on the set $\{1,2, \ldots, n\}$;

(ii) $\check{G}_{b}$ is totally strong self-complement if it is identical to $\check{G}_{b}^{\phi c}$, the $\phi$-complement of $\check{G}_{b}$, for all permutations $\phi$ on the set $\{1,2, \ldots, n\}$.

Example 48. All strong BFGSs are the only examples of selfcomplement or totally self-complement BFGSs.

Example 49. A BFGS $\check{G}_{b}=\left(M, N_{1}, N_{2}, N_{3}\right)$ of graph structure $G^{*}=\left(U, E_{1}, E_{2}, E_{3}\right)$ as shown in Figure 10 is totally strong self-complement.

Theorem 50. A BFGS $\check{G}_{b}$ is strong if and only if it is totally self-complement.

Proof. Let $\check{G}_{b}$ be a strong BFGS and $\phi$ any permutation on the set $\{1,2, \ldots, n\}$.

By Theorem 45, $\check{G}_{b}^{\phi c}$ is strong and if $\phi^{-1}(i)=j$, then all $N_{i}$-edges in $\check{G}_{b}=\left(M, N_{1}, N_{2}, \ldots, N_{n}\right)$ become $N_{j}^{\phi}$-edges in $\check{G}_{b}^{\phi c}=\left(M, N_{1}^{\phi}, N_{2}^{\phi}, \ldots, N_{n}^{\phi}\right)$

$$
\begin{aligned}
\Longrightarrow & \mu_{N_{i}}^{P}\left(a_{1} a_{2}\right)=\mu_{M}^{P}\left(a_{1}\right) \wedge \mu_{M}^{P}\left(a_{2}\right) \\
& =\mu_{N_{j}^{\phi}}^{P}\left(a_{1} a_{2}\right), \\
\mu_{N_{i}}^{N}\left(a_{1} a_{2}\right) & =\mu_{M}^{N}\left(a_{1}\right) \vee \mu_{M}^{N}\left(a_{2}\right)=\mu_{N_{j}^{\phi}}^{N}\left(a_{1} a_{2}\right) .
\end{aligned}
$$

Hence $\check{G}_{b}$ is isomorphic to $\check{G}_{b}^{\phi c}$ under the identity mapping $f: U \rightarrow U$, such that $\mu_{M}^{P}(a)=\mu_{M}^{P}(f(a)), \mu_{M}^{N}(a)=$ $\mu_{M}^{N}(f(a)) \forall a \in U$ and

$$
\begin{array}{r}
\mu_{N_{i}}^{P}\left(a_{1} a_{2}\right)=\mu_{N_{j}^{\phi}}^{P}\left(a_{1} a_{2}\right)=\mu_{N_{j}^{\phi}}^{P}\left(f\left(a_{1}\right) f\left(a_{2}\right)\right), \\
\mu_{N_{i}}^{N}\left(a_{1} a_{2}\right)=\mu_{N_{j}^{\phi}}^{N}\left(a_{1} a_{2}\right)=\mu_{N_{j}^{\phi}}^{N}\left(f\left(a_{1}\right) f\left(a_{2}\right)\right), \\
\forall a_{1} a_{2} \in E_{i},
\end{array}
$$

for $\phi^{-1}(i)=j, i, j=1,2, \ldots, n$. This holds for any permutation on the set $\{1,2, \ldots, n\}$.

Hence $\check{G}_{b}$ is totally self-complement.

Conversely, let $\check{G}_{b}$ and $\check{G}_{b}^{\phi c}$ be isomorphic for any permutation $\phi$ on the set $\{1,2, \ldots, n\}$. Then from the definition of $\phi$-complement and isomorphism of BFGSs, we have

$$
\begin{aligned}
\mu_{N_{i}}^{P}\left(a_{1} a_{2}\right) & =\mu_{N_{j}^{\phi}}^{P}\left(f\left(a_{1}\right) f\left(a_{2}\right)\right) \\
& =\mu_{M}^{P}\left(f\left(a_{1}\right)\right) \wedge \mu_{M}^{P}\left(f\left(a_{2}\right)\right) \\
& =\mu_{M}^{P}\left(a_{1}\right) \wedge \mu_{M}^{P}\left(a_{2}\right), \\
\mu_{N_{i}}^{N}\left(a_{1} a_{2}\right) & =\mu_{N_{j}^{\phi}}^{N}\left(f\left(a_{1}\right) f\left(a_{2}\right)\right) \\
& =\mu_{M}^{N}\left(f\left(a_{1}\right)\right) \vee \mu_{M}^{N}\left(f\left(a_{2}\right)\right) \\
& =\mu_{M}^{N}\left(a_{1}\right) \vee \mu_{M}^{N}\left(a_{2}\right)
\end{aligned}
$$

$\forall a_{1} a_{2} \in E_{i}, i=1,2, \ldots, n$.

Hence, $\check{G}_{b}$ is a strong BFGS.

Remark 51. Every self-complement BFGS is necessarily totally self-complement.

Theorem 52. If graph structure $G^{*}=\left(U, E_{1}, E_{2}, \ldots, E_{n}\right)$ is totally strong self-complement and $M=\left(\mu_{M}^{P}, \mu_{M}^{N}\right)$ is a bipolar fuzzy set of $U$ with constant valued functions $\mu_{M}^{P}$ and $\mu_{M}^{N}$, then a strong $B F G S \check{G}_{b}=\left(M, N_{1}, N_{2}, \ldots, N_{n}\right)$ of $G^{*}$ is totally strong self-complement.

Proof. Let $s \in[0,1]$ and $t \in[-1,0]$ be two constants, such that

$$
\begin{aligned}
& \mu_{M}^{P}(u)=s, \\
& \mu_{M}^{N}(u)=t
\end{aligned}
$$

$\forall u \in U$

Since $G^{*}$ is totally strong self-complement, so for every permutation $\phi^{-1}$ on the set $\{1,2, \ldots, n\}$, there exists a bijection $f$ : $U \rightarrow U$, such that for every $E_{i}$-edge $a_{1} a_{2}$, " $f\left(a_{1}\right) f\left(a_{2}\right)$ " [an $E_{j}$-edge in $\left.G^{*}\right]$ is an $E_{i}$-edge in $\left(G^{*}\right)^{\phi^{-1} c}$ and, consequently, 
for every $N_{i}$-edge $a_{1} a_{2}$, " $f\left(a_{1}\right) f\left(a_{2}\right)$ " [a $N_{j}$-edge in $\check{G}_{b}$ ] is a $B_{i}^{\phi}$-edge in $\check{G}_{b}^{\phi c}$. Moreover $\check{G}_{b}$ is strong, so we have

$$
\begin{aligned}
\mu_{M}^{P}(a) & =s=\mu_{M}^{P}(f(a)), \\
\mu_{M}^{N}(a) & =t=\mu_{M}^{N}(f(a)) \\
\mu_{N_{i}}^{P}\left(a_{1} a_{2}\right) & =\mu_{M}^{P}\left(a_{1}\right) \wedge \mu_{M}^{P}\left(a_{2}\right) \\
& =\mu_{M}^{P}\left(f\left(a_{1}\right)\right) \wedge \mu_{M}^{P}\left(f\left(a_{2}\right)\right) \\
& =\mu_{N_{j}^{\phi}}^{P}\left(f\left(a_{1}\right) f\left(a_{2}\right)\right), \\
\mu_{N_{i}}^{N}\left(a_{1} a_{2}\right) & =\mu_{M}^{N}\left(a_{1}\right) \vee \mu_{M}^{N}\left(a_{2}\right) \\
& =\mu_{M}^{N}\left(f\left(a_{1}\right)\right) \vee \mu_{M}^{N}\left(f\left(a_{2}\right)\right) \\
& =\mu_{N_{j}^{\phi}}^{N}\left(f\left(a_{1}\right) f\left(a_{2}\right)\right)
\end{aligned}
$$

$\forall a_{1} a_{2} \in E_{i}, \quad i=1,2, \ldots, n$.

This shows that $\check{G}_{b}$ is strong self-complement. This holds for any permutation $\phi$ and $\phi^{-1}$ on the set $\{1,2, \ldots, n\}$; thus $\check{G}_{b}$ is totally strong self-complement. This completes the proof.

Remark 53. The converse of Theorem 52 is not necessary, since a totally strong self-complement BFGS $\check{G}_{b}=$ $\left(M, N_{1}, N_{2}, N_{3}\right)$, as shown in Figure 10, is strong and has a totally strong self-complement underlying graph structure, but $\mu_{M}^{P}$ and $\mu_{M}^{N}$ are not constant valued functions.

\section{Conclusions}

Graph-theoretical concepts are widely used to study and model various applications in different areas. However, in many cases, some aspects of a graph-theoretical problem may be vague or uncertain. It is natural to deal with the vagueness and uncertainty using the methods of fuzzy sets. Since bipolar fuzzy set has shown advantages in handling vagueness and uncertainty than fuzzy set, we have applied the concept of bipolar fuzzy sets to graph structures. We have introduced the concept of bipolar fuzzy graph structures. We are extending our work to (1) bipolar fuzzy soft graph structures, (2) soft graph structures, (3) rough fuzzy soft graph structures, and (4) roughness in fuzzy graph structures.

\section{Conflict of Interests}

The authors declare that there is no conflict of interests regarding the publication of this paper.

\section{Acknowledgment}

The authors are thankful to the anonymous referees for the critical review of their paper.

\section{References}

[1] E. Sampathkumar, "Generalized graph structures," Bulletin of Kerala Mathematics Association, vol. 3, no. 2, pp. 65-123, 2006.

[2] L. A. Zadeh, "Fuzzy sets," Information and Computation, vol. 8, pp. 338-353, 1965.

[3] W.-R. Zhang, "(Yin) (Yang) bipolar fuzzy sets," in Proceedings of the IEEE International Conference on Fuzzy Systems Proceedings and the IEEE World Congress on Computational Intelligence (FUZZ-IEEE '98), vol. 1, pp. 835-840, IEEE, Anchorage, Alaska, USA, May 1998.

[4] A. Kauffman, Introduction a la Theorie des Sous-Emsembles Flous, vol. 1, Masson et Cie, 1973.

[5] A. Rosenfeld, "Fuzzy graphs," in Fuzzy Sets and Their Applications, L. A. Zadeh, K. S. Fu, and M. Shimura, Eds., pp. 77-95, Academic Press, New York, NY, USA, 1975.

[6] P. Bhattacharya, "Some remarks on fuzzy graphs," Pattern Recognition Letters, vol. 6, no. 5, pp. 297-302, 1987.

[7] J. N. Mordeson, P. S. Nair, and J. N. Mordeson, Fuzzy Graphs and Fuzzy Hypergraphs, Second Edition 2001, Physica, Heidelberg, Germany, 2nd edition, 1998.

[8] T. Dinesh, A study on graph structures, incidence algebras and their fuzzy analogues [Ph.D. thesis], Kannur University, Kannur, India, 2011.

[9] M. Akram, "Bipolar fuzzy graphs," Information Sciences, vol. 181, no. 24, pp. 5548-5564, 2011.

[10] M. Akram, "Bipolar fuzzy graphs with applications," KnowledgeBased Systems, vol. 39, pp. 1-8, 2013.

[11] M. Akram and W. A. Dudek, "Regular bipolar fuzzy graphs," Neural Computing \& Applications, vol. 21, no. 1, pp. 197-205, 2012.

[12] M. Akram, S.-G. Li, and K. P. Shum, "Antipodal bipolar fuzzy graphs," Italian Journal of Pure and Applied Mathematics, vol. 31, pp. 97-110, 2013.

[13] M. Akram, W. A. Dudek, and S. Sarwar, "Properties of bipolar fuzzy hypergraphs," Italian Journal of Pure and Applied Mathematics, vol. 31, pp. 426-458, 2013.

[14] N. O. Al-Shehrie and M. Akram, "Bipolar fuzzy competition graphs," Ars Combinatoria, vol. 121, pp. 385-402, 2015.

[15] K.-M. Lee, "Bipolar-valued fuzzy sets and their basic operations," in Proceedings of the International Conference, pp. 307317, Bangkok, Thailand, 2000.

[16] S. Mathew and M. S. Sunitha, "Node connectivity and arc connectivity of a fuzzy graph," Information Sciences, vol. 180, no. 4, pp. 519-531, 2010.

[17] L. A. Zadeh, "Similarity relations and fuzzy orderings," Information Sciences, vol. 3, no. 2, pp. 177-200, 1971.

[18] W.-R. Zhang, "Bipolar fuzzy sets and relations: a computational framework forcognitive modeling and multiagent decision analysis," in Proceedings of the Industrial Fuzzy Control and Intelligent Systems Conference, and the NASA Joint Technology Workshop on Neural Networks and Fuzzy Logic, Fuzzy Information Processing Society Biannual Conference, pp. 305-309, San Antonio, Tex, USA, December 2014. 

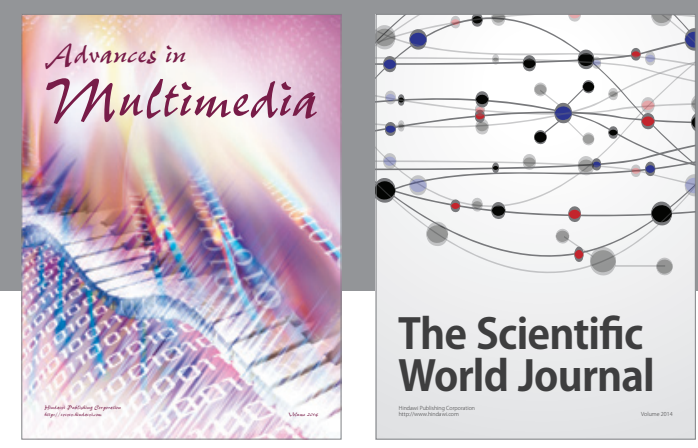

The Scientific World Journal
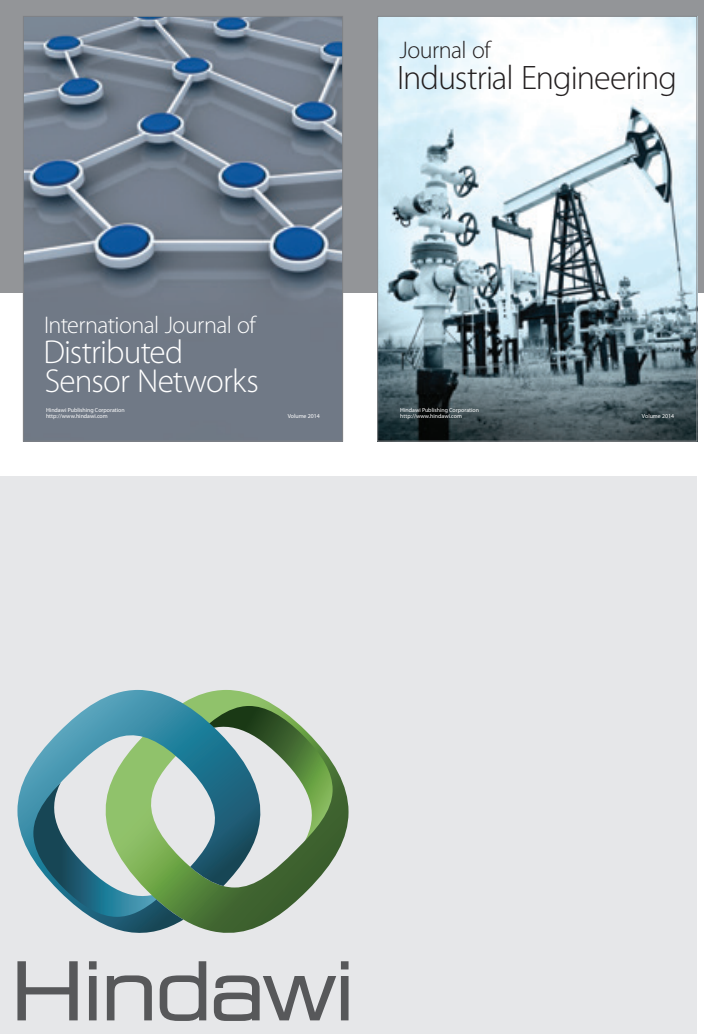

Submit your manuscripts at

http://www.hindawi.com

\section{Computer Networks} and Communications
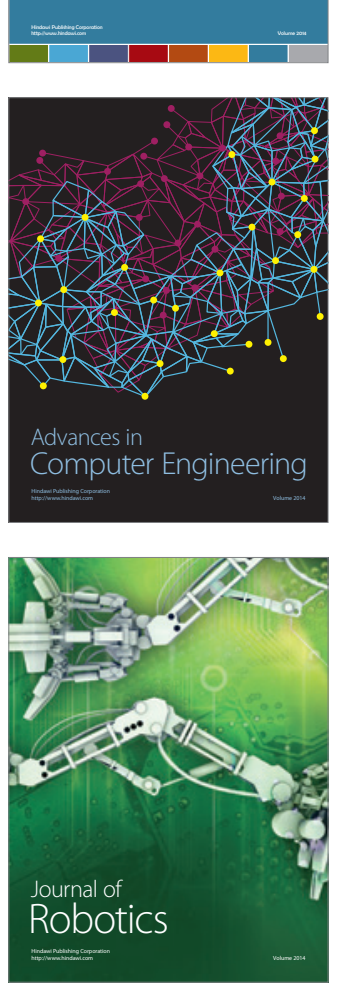
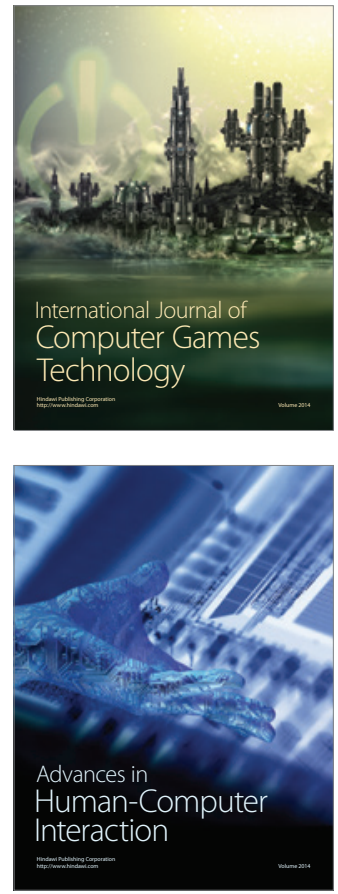
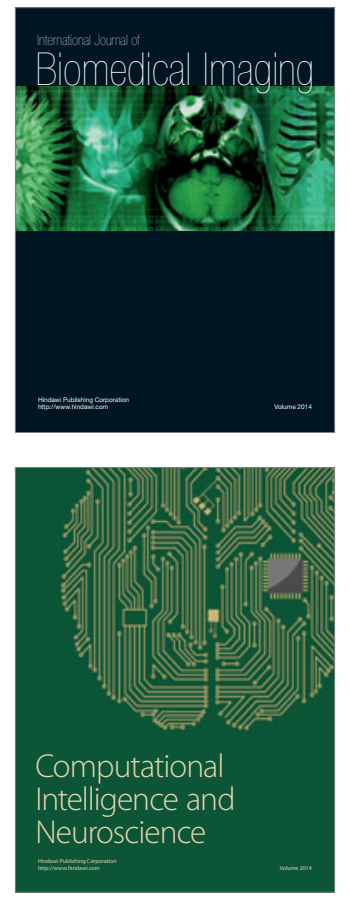
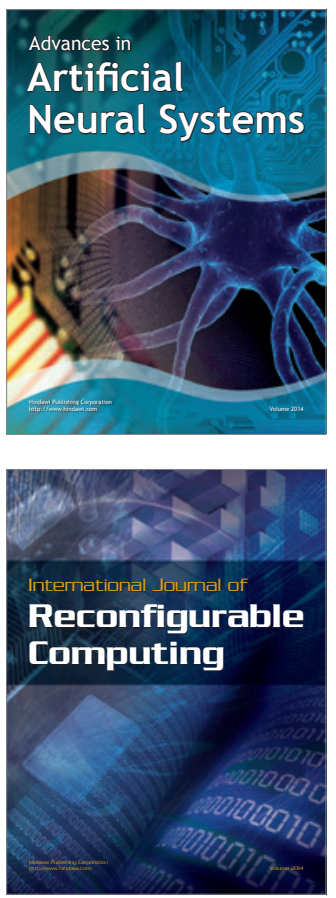
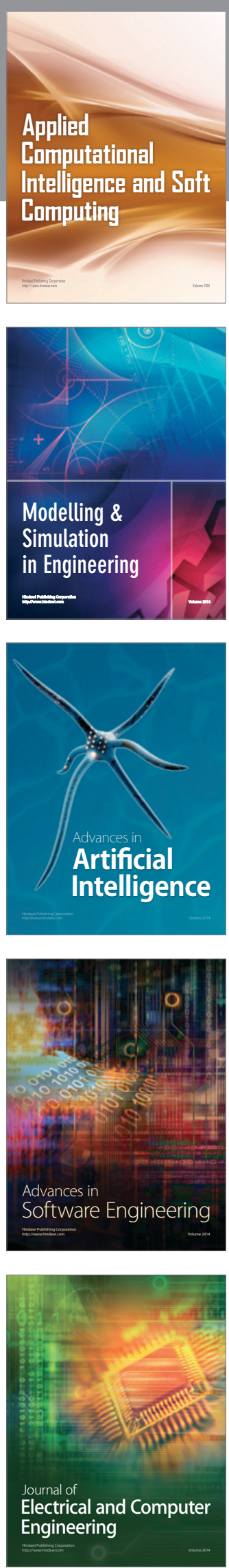